\title{
Una città in fermento. Gli intellettuali napoletani e il ruolo del viceré Francisco de Benavides, IX conte di Santisteban (I688-I696)
}

\author{
Paola Setaro \\ Universidad Autónoma de Madrid
}

Una ciutat en ferment. Els intel.lectuals napolitans $i$ el paper del virrei Francisco de Benavides, IX comte de Santisteban (I688-1696)

\section{Resum}

Aquest treball se centra en la renovació cultural de Nàpols els últims anys del segle XVII, en particular en la relació que s'estableix entre cultura i vida civil. Es perfila la posició que assumeix Francisco de Benavides, virrei a Nàpols del i688 al I696, en la llarga i complicada polèmica anticurial, a través de la defensa de la filosofia moderna professada pels membres de l'anomenada Acadèmia dels Investiganti, especialment Francesco d'Andrea i Giuseppe Valletta. El virrei no només va freqüentar assíduament la riquíssima biblioteca de Giuseppe Valletta, sinó que va defensar Francesco d'Andrea d'algunes acusacions injustes. Unes cartes, conservades a la seu toledana de l'Arxiu Ducal de Medinaceli i fins ara inèdites, entre el virrei, el secretari espanyol Manuel Francisco de Lira, el president del Sacre Regio Consell Feliç de Lanzina i Ulloa i el regent de l'Collateral Diego Sòria de Morales, són un testimoni de la difícil paràbola política de D'Andrea i destaquen el suport que li va oferir Benavides.

Paraules clau: Nàpols, finals del segle XviI, Acadèmia dels Investiganti, ateisme, Francisco de Benavides, Giuseppe Valletta, Francesco d'Andrea. 
Una ciudad en fermento. Los intelectuales napolitanos y el papel del virrey Francisco de Benavides, IX conde di Santisteban (I688-1696)

\title{
Resumen
}

Este trabajo se centra en la renovación cultural de Nápoles durante los últimos años del siglo XVII, en particular en la relación que se establece entre cultura y vida civil. Se perfila la posición que asume Francisco de Benavides, virrey en Nápoles de I688 a i696, en la larga y complicada polémica anticurial, a través de la defensa de la filosofía moderna profesada por los miembros de la Academia de los Investiganti, especialmente Francesco D’Andrea y Giuseppe Valletta. El virrey no solo frecuentó asiduamente la riquísima biblioteca de Giuseppe Valletta, sino que defendió a Francisco D’Andrea de algunas acusaciones injustas. Algunas cartas, conservadas en la sede toledana del Archivo Ducal de Medinaceli y hasta ahora inéditas, entre el virrey, el secretario español Manuel Francisco de Lira, el presidente del Sacro Regio Consejo Feliz de Lanzina y Ulloa y el regente del Collaterale Diego Soria de Morales, son un testimonio de la difícil parábola política de D’Andrea y destacan el apoyo que le ofreció Benavides al reclutarlo en los despachos de la burocracia napolitana.

Palabras clave: Nápoles, finales del siglo xvir, Academia de los Investiganti, ateísmo, Francisco de Benavides, Giuseppe Valletta, Francesco D’Andrea.

\section{A city in ferment. Neapolitan intellectuals and the role of the Viceroy Francisco de Benavides, 9th Count of Santisteban (1688-1696)}

\begin{abstract}
This paper focuses on the cultural renewal in Naples during the last years of the i7th century, and in particular on the relationship between culture and civil life. It outlines the position taken by Francisco de Benavides, viceroy in Naples from I688 to 1696, in the long and complicated anticurial polemic, through the defense of the modern philosophy professed by the members of the Accademia degli Investiganti, especially Francesco D’Andrea and Giuseppe Valletta. The viceroy not only frequented Giuseppe Valletta's very rich library assiduously, but also defended Francesco D'Andrea against some unjust accusations. Some letters between the viceroy, the Spanish secretary Manuel Francisco de Lira, the president of the Sacred Royal Council Feliz de
\end{abstract}


Lanzina y Ulloa and the regent of the Colateral Diego Soria de Morales, preserved in the Toledo seat of the Ducal Archives of Medinaceli and hitherto unpublished, bear witness to D'Andrea's difficult political parable and highlight the support Benavides offered him in recruiting him to the offices of the Neapolitan bureaucracy.

Keywords: Naples, late I7th century, Accademia degli Investiganti, atheism, Francisco de Benavides, Giuseppe Valletta, Francesco D’Andrea.

Se la tematica delle politiche artistiche messe in atto dai viceré a Napoli nel XVII secolo, unitamente alle vicende individuali e alle traiettorie delle loro carriere, sono diventate negli ultimi vent'anni oggetto di ricerca di numerose tesi di dottorato, non si può dire lo stesso, a parte poche eccezioni, per quanto riguarda il loro rapporto con il mondo degli intellettuali. Da questo punto di vista, per l'ampiezza della trattazione e per i numerosi spunti di riflessioni offerti, si ricordano le tesi di Isabel Enciso Alonso-Munumer, Nobleza, poder y mecenazgo en tiempos de Felipe III. Nápoles y el conde de Lemos, del 2002, di Ana Minguito Palomares, Linaje, poder y cultura: el gobierno de Ínigo Vélez de Guevara, VIII conde de Oñate en Nápoles (I648-I653), in cui si affrontano i percorsi dei viceré da un punto di vista tripartito - origini, politica e mecenatismo - e di Felipe Vidales del Castillo, El VII Marqués del Carpio $y$ las letras. Quest'ultima, in cui si ricostruiscono origini, contenuto e soprattutto finalità delle collezioni librarie del marchese del Carpio, può considerarsi il primo lavoro in cui la biografia di un viceré è ricostruita da una prospettiva esclusivamente culturale. ${ }^{\text {I }}$ Ma se questa ha

I. Rispettivamente, Isabel Enciso Alonso-Muñumer, «Nobleza, poder y mecenazgo en tiempos de Felipe III. Nápoles y el Conde de Lemos», Universidad Complutense de Madrid, 2002; Ana Minguito Palomares, «Linaje, poder y cultura: el gobierno de Íñigo Vélez de Guevara, VIII conde de Oñate en Nápoles (I648-I653)», Universidad Complutense de Madrid, 200I (poi pubblicata come monografia: Nápoles y el virrey conde de Oñate. La estrategia del poder y el resurgir del reino (I648-I653), Sílex, Madrid, 20II); Felipe Vidales del Castillo, «El VII Marqués del Carpio y las letras», Universidad Complutense de Madrid, 2016. 
riempito un vuoto sul rapporto che legava il viceré del Carpio al mondo degli editori, librai e stampatori, manca ancora una messa fuoco complessiva del rapporto tra i viceré e gli intellettuali legati alle accademie, spazi di grande vitalità che conobbero una forte crescita negli ultimi decenni del XVII secolo, prima fra tutte l'Accademia degli Oziosi. ${ }^{2}$

In questo senso si può considerare esemplificativo un passo di Giambattista Marino, che nel I628 scriveva che «il Sig. Vicerè è quasi ogni giorno meco; mi fa favori non ordinari, e dimostra di compiacersi della mia conversatione».3 Il poeta si riferisce alle frequenti visite da parte di Antonio Álvarez de Toledo, V duca d'Alba e viceré di Napoli dal I622 al I629, e alla sua nomina a principe dell'Accademia degli Oziosi. Quest'ultima, fondata nel I6II da Giambattista Manso insieme a Pedro Fernández de Castro, viı conte di Lemos e viceré di Napoli, radunò i più importanti intellettuali dell'epoca, napoletani e spagnoli, proponendo scambi con le altre accademie d'Italia. ${ }^{4}$ Il rapporto tra il viceré e l'istituzione degli Oziosi, dove si discuteva di poetica, retorica, matematica e filosofia, escludendo (almeno apparentemente) in maniera rigorosa temi politici e teologici, è stato affrontato ampiamente da Isabel Enciso Alonso-Muñumer. ${ }^{5}$ Dedicatario delle principali opere del Cervantes maturo e di testi di autori napoletani tra cui spicca Giovan

2. Sul tema della stampa come veicolo di rappresentazione del potere da parte dei viceré, si rimanda a Encarnación SÁnchez García, Imprenta y cultura en la Nápoles virreinal: los signos de la presencia española, Alinea, Firenze, 2007, in cui la studiosa mette in luce l'impronta ideologica lasciata dalla politica vicereale nella produzione libraria napoletana tra il XVI e il XVII secolo, e EADEM, "Aplicossi a render immortale la sua memoria nel Regno". El virrey Medina de las Torres en Nápoles», in A. Carrasco Martínez, a cura di, La nobleza y los reinos. Anatomía del poder en la Monarquía de España (siglos XVI-XVII), Iberoamericana-Vervuert, Madrid-Francoforte, 20I7, pp. 36I-394.

3. Giambattista Marino, Lettere Gravi, Argute e Facete. Non più Stampate. Con alcune Poesie dell'Istesso, Giacomo Sarzina, Venezia, 1628, p. 72.

4. Sulle pratiche dell'Accademia si rimanda a Girolamo De Miranda, Una quiete operosa. Forma e pratiche dell'Accademia napoletana degli Oziosi, I6II-I645, Fridericiana Editrice Universitaria, Napoli, 2000.

5. Isabel Enciso Alonso-Muñumer, Nobleza, poder y mecenazgo en tiempos de Felipe III. Nápoles y el Conde de Lemos, Actas, Madrid, 2007.

Pedralbes, 4I (2O2I), 255-298, ISSN: O2II-9587, DOI: IO.344/PEDRALBES2O2I.4I.8 
Battista Della Porta, Pedro Fernández de Castro costituì il fulcro di un poderoso circolo culturale e letterario, che si servì a sua volta della formula della propaganda per esaltare l'importanza della propria cultura. ${ }^{6}$ Questo lavoro sul conte di Lemos offre una riflessione sulle diverse linee di interpretazione che prendono avvio da un interrogativo fondamentale: in che misura incisero le iniziative del potere vicereale nell'organizzazione del nucleo intellettuale e delle sue scelte? Il dibattito storiografico è ormai generalmente orientato verso l'idea della creazione di un modello aristocratico di cultura attraverso il sodalizio tra potere spagnolo e intellettuali, e non di semplice emanazione del potere vicereale. ${ }^{7}$ E se è vero che spesso l'intellettuale si muoveva tra la dimensione letteraria e l'ambizione a ricoprire importanti cariche amministrative e politiche grazie alla protezione del viceré, è anche vero che la scelta dei temi e il funzionamento delle accademie non derivarono tutte dalle direttive del governante.

Anche nel lavoro di Ana Minguito Palomares su Íñigo Vélez de Guevara, VIII conte di Ońate, che governò dal I648 al I653, in una fase molto delicata come quella post-rivoluzionaria, si affronta il tema dalla medesima prospettiva. Il conte di Ońate non si tenne al margine della vita delle accademie, ma anzi continuò a tenere viva quella degli Oziosi, a cui partecipava attivamente, così come promosse la nascita, nel I648, dell'Accademia dei Rinnovati, dove si discuteva di temi giuridici. ${ }^{8}$ Erano gli anni in cui la monarchia spagnola aveva dato avvio a una politica di riforme a livello istituzionale e a un preciso progetto politico, che pre-

6. Si veda Girolamo De Miranda, «Il viceré letterato. Mecenatismo artistico e religioso di Pedro Fernandez de Castro tra Madrid, Napoli e Monforte de Lemos», in J. Luis Colomer, a cura di, España y Nápoles. Coleccionismo y mecenazgo virreinales en el siglo XVII, Centro de Estudios Europa Hispánica, Madrid, 2009, pp. 215-227.

7. Nel saggio di Anna Maria RaO, «Fra amministrazione e politica. Gli ambienti intellettuali napoletani», in J. Boutier, B. Marine, A. Romano, a cura di, Naples, Rome, Florence. Une histoire comparée des milieux intellectuels italiens (XVII-XVIIIè siècles), Publications de l'École française de Rome, Roma, 2005, pp. 35-88, si fa accenno a questo interessante dibattito storiografico.

8. Ana Minguito Palomares, Nápoles y el virrey conde de Oñate. La estrategia del poder y el resurgir del reino (I648-I653), Sílex, Madrid, $201 \mathrm{I}$. 
vedeva una svolta anche per la vita intellettuale, e fu in questo frangente che la vita civile fu sostenuta da una cultura nuova. ${ }^{9}$ Quella precedente alla rivolta antispagnola era stata caratterizzata dall'intransigente scolasticismo delle filosofie controriformistiche, da cui dipendevano l'economia, il diritto e la medicina. Il sintomo dell'entrata in crisi di questo sistema fu rappresentato dall'Accademia fondata nel 1645 da Camillo Colonna, detta Colonnese o anche delle Mortelle..$^{10}$ Nata dopo la rivoluzione masanelliana ed estinta già prima del 1657 , attrasse la giovane intellighentia napoletana ed è considerata tra le prime istituzioni responsabili dell'apertura del canale di comunicazione fra Napoli e l'Europa. ${ }^{\text {II }}$ Questo primo tentativo di applicare il metodo empirico alla filosofia procedette molto lentamente, ostacolato dall'opposizione dei detrattori che dominavano nella scienza ufficiale, dalle minacce dell'Inquisizione, ma anche dall'epidemia di peste del 1656, che diede una battuta d'arresto alle riunioni, così come agli esperimenti che si stavano conducendo in città. ${ }^{12}$

9. Su questa fase si veda il lavoro di Pier Luigi Rovito, Il viceregno spagnolo di Napoli, Arte Tipografica, Napoli, 2003. Sulle rivolte degli anni Quaranta in Catalogna, Portogallo, Sicilia e sui viceré protagonisti, si veda Aurelio Musi, L'impero dei viceré, Il Mulino, Bologna, 20I3 pp. I92-208. Per un affondo sul contesto culturale e la nascita di un marcato impegno civile nella seconda metà del xvir secolo, due testi imprescindibili sono: Salvo Mastellone, Pensiero politico e vita culturale a $\mathrm{Na}$ poli nella seconda metà del Seicento, D’Anna, Firenze, 1965, e Giuseppe Galasso, Napoli spagnola dopo Masaniello, Edizioni di Storia e Letteratura, Roma, 2019 (prima edizione: Edizioni Scientifiche Italiane, Napoli, 1972), in particolare pp. 86-I2O.

Io. Le riunioni si tenevano nella sua dimora napoletana ubicata nella strada di San Carlo delle Mortelle: da qui la derivazione del nome dell'Accademia; la notizia è in Augusto De Ferrari, s.v. "Colonna, Camillo», in Dizionario Biografico degli Italiani, Istituto dell'Enciclopedia Italiana, Roma, 1982, vol. 27.

II. Biagio De Giovanni, «La vita intellettuale a Napoli fra la metà del '6oo e la restaurazione del Regno", in Storia di Napoli, Società Editrice Storia di Napoli, Napoli, 1970, vol. 6 (Tra Spagna e Austria), pp. 443-464.

I2. Il dibattito sulla natura della peste, che aveva assorbito numerosi medici e scienziati, tra cui Tommaso Cornelio, era giunto alla conclusione che la malattia poteva trasmettersi anche solo con la vicinanza agli appestati e che l'unico rimedio preventivo consisteva nell'isolamento. La dimostrazione scientifica di quest'ultimo 
Nel 1649 aveva deciso di trasferirsi definitivamente a Napoli, dopo un viaggio in Italia, il filosofo, medico e matematico di origine calabrese Tommaso Cornelio, che costituì un fondamentale punto di riferimento per gli studi medico-filosofici, grazie alla sua diffusione delle idee di Gassendi, di Cartesio, di Bayle, di Grozio e di altri filosofi del tempo. ${ }^{13}$ Il suo nome è soprattutto legato alla creazione dell'Accademia degli Investiganti, patrocinata da Andrea Conclubert, marchese di Arena; questa istituzione si propose di confutare, in nome della ragione cartesiana, ogni tipo di indagine basata sulla vecchia autorità di Aristotele. ${ }^{14}$ I suoi membri furono espressione del cosiddetto ceto civile, cioè la nuova borghesia composta da letterati, magistrati, fisici, medici, ma soprattutto avvocati, orientata verso un serrato impegno sociale. I suoi componenti condivisero principalmente l'aspirazione a ricoprire un ruolo attivo nella burocrazia napoletana e si resero protagonisti della lotta anticuriale. ${ }^{\text {is }} \mathrm{Fu}$ sotto il governo del viceré Monterrey (I63I-I637) che si rese evidente quel processo che avrebbe sempre di più caratterizzato la vita cittadina nel corso del xviı secolo, incarnato nell'opportunità, non solo per gli aristocratici, ma anche per burocrati e giuristi, i cosiddetti togati, di ricoprire cariche pubbliche nelle principali magistrature: il Collaterale, il Sacro Regio Consiglio, la Sommaria e la Vicaria. ${ }^{16}$ Il fenomeno si rese evidente durante il viceregno di Pedro de

punto si deve al medico Geronimo Gatta, originario di Sala Consilina, che nel suo trattato analizza il morbo da una prospettiva nuova, avvicinandosi così a quel settore di intellettuali convinto dell'importanza di teorizzare a partire sempre dall'esperienza; sul testo di Gatta, sulle sue fonti e sugli elementi di innovazione contenuti in esso, si veda il saggio di Silvana D’Alessio, "L'aria innocente. Geronimo Gatta e le sue fonti», Mediterranea-ricerche storiche, 44 (dicembre 2018), pp. 587-6I2.

I3. Vittorio Ivo Comparato, s.v. "Cornelio, Tommaso", in Dizionario Biografico degli Italiani, Istituto dell'Enciclopedia Italiana, Roma, 1983, vol. 29, ad vocem.

I4. Sull'Accademia degli Investiganti si veda l'insuperabile testo di Max H. Fisch, «L'Accademia degli Investiganti», De Homine, 27-28 (dicembre 1968), pp. 17-68.

I5. Giovanni Muto, "Gestione politica e controllo sociale nella Napoli spagnola», in M. Bosse, A. Stoll, a cura di, Napoli viceregno spagnolo. Una capitale della cultura alle origini dell'Europa moderna (sec. XVI-XVII), Vivarium, Napoli, 200I, vol. I, pp. 65-IOO.

16. Per un'elenco prosopografico delle magistrature, un utilissimo strumento è il minuzioso lavoro di Gaetana Intorcia, Magistrature del Regno di Napoli. Analisi 
Aragón (I666-I67I) ma raggiunse un momento di grande intensità negli anni napoletani di Fernando Fajardo y Álvarez de Toledo, marchese di Los Vélez (1675-1683). Fu però solo grazie alla politica vicereale inaugurata dal marchese del Carpio (I683-1687), che il ceto civile assunse un ruolo di primo piano nell'amministrazione. Ebbe così inizio un processo inarrestabile che porterà "l'accentuato impegno civile ${ }^{17}$ a incidere sempre di più nella vita cittadina. L'assetto dei togati si consolidò con l'avvento a Napoli del viceré Benavides (I688-1696), grazie al sostegno del progetto di riforma della classe dirigente napoletana intrapreso dall'avvocato Francesco D'Andrea, uno degli intellettuali più lucidi della seconda metà del XviI secolo, fautore del risveglio culturale partenopeo.

\section{L'Accademia degli Investiganti}

Il vescovo e storico inglese Gilbert Burnet, in viaggio a Napoli negli anni del viceregno del marchese del Carpio, evidenziava che la città era permeata da un'estrema contraddizione: da un lato la persistenza di pratiche superstiziose, dall'altro la presenza di «uomini di più libero pensiero che in ogni altra parte d'Italia». ${ }^{18}$ Il libero pensiero di cui parla Burnet è quello dell'Accademia degli Investiganti, di cui fecero parte molti intellettuali, come Carlo Buragna, Tommaso Cornelio, Francesco D’Andrea, Leonardo Di Capua, Sebastiano Bartoli, Lucantonio Porzio, Giuseppe Valletta e Nicola Caravita. Fu un'esperienza molto

prosopografica. Secoli XVI-XVII, Jovene editore, Napoli, 1987. Sull'ascesa del ceto forense nella Napoli del xvi secolo, analizzata nel più ampio contesto del panorama giuridico e politico italiano, si veda Pierluigi Rovito, La Respublica dei togati. Giuristi e società nella Napoli del Seicento, Jovene editore, Napoli, I98I.

17. Giuseppe GaLasso, «Napoli nel viceregno spagnolo (1696-1707)», in Storia di Napoli. Fra Spagna e Austria, Società Editrice Storia di Napoli, Napoli, 1970, vol. 6 (Tra Spagna e Austria), p. 3.

I8. Gilbert BuRnet, Burnet's Travels or, a collection of letters to the Hon. Robert Boyle, printed for and sold by Ward and Chandler, London, I738, p. I6o (traduzione dell'autore). 
intensa, fatta di arresti e rinascite e che culminò in una vera e propria lotta contro la cultura aristotelica e scolastica. ${ }^{19}$

Nata formalmente nel I663, quando il padre benedettino Juan Caramuel y Lóbkowitz, vescovo di Campagna dal I657 al I673, affermò che l'Accademia era stata chiamata così per ricercare «la verità latente nella Natura», ${ }^{20}$ in realtà questa era già attiva dalla fine del I649, quando alcuni intellettuali si riunivano a casa di Cornelio per leggere una selezione di testi dei maggiori pensatori europei contemporanei. Fu soprattutto l'avvocato Francesco D'Andrea a riconoscere per primo la portata innovativa della lezione di Cornelio, come afferma egli stesso in una pagina di $A v$ vertimenti ai nipoti, scritto nel 1696 e dedicato ai figli del fratello Gennaro, con la speranza che continuassero la strada dell'avvocatura:

[...] onde, venuto in Napoli l'anno I649 il nostro signor Tommaso Cornelio a cui la nostra città deve tutto ciò oggi si sa di più verisimile nella filosofia e nella medicina, io fui il primo che abbracciassi quella maniera da lui propostaci di filosofare con far venire in Napoli l'opera di Renato delle Carte [Descartes], di cui sino a quel tempo n'era stato a noi incognito il nome. ${ }^{21}$

I9. Maurizio Torrini, «L'Accademia degli Investiganti. I663-I670», Quaderni storici, 48 (198I), pp. 845-883; IDEM, «Scienza e filosofia nell'Italia del Seicento», in M. Tedeschi, a cura di, Il Mezzogiorno a Napoli nel Seicento italiano, Soveria Mannelli, Rubettino, 2003, pp. 9I-IO2.

20. La citazione è riportata da Fisch, L'Accademia degli Investiganti, p. 24. Il periodo napoletano di Caramuel, uno dei più importanti membri dell'Accademia, arrivato nella città partenopea per dirimere alcune controversie di tipo giudiziario, è poco documentato; la notizia è riportata in Ida MaUro, Spazio urbano e rappresentazione del potere: le cerimonie della città di Napoli dopo la rivolta di Masaniello (I648I672), Federico II University Press, Napoli, 2020, p. 216. Su Caramuel il contributo più recente è di Jorge Fernández-Santos Ortiz-Iribas, "La única pluma de que se valen los ministros de Vuestra Magestad en Italia': Juan Caramuel ante los esfuerzos de renovación política y cultural en el reinado de Carlos II», in D. Sabaino, P. C. Pissavino, a cura di, Un'altra modernità. Juan Caramuel (I606-I682): enciclopedia e probabilismo, Edizioni ETS, Pisa, 20I2, pp. 25-83.

2I. Francesco D'Andrea, Avvertimenti ai nipoti, a cura di Imma Ascione, Jovene, Napoli, I990, p. 203. 
Francesco D'Andrea, che già grazie a Camillo Colonna aveva conosciuto una filosofia «non molto dissimile da quella che oggi chiamiamo atomista», ${ }^{22}$ fu una figura chiave del gruppo degli Investiganti ma anche uno dei più noti avvocati d'Italia, come metteva in luce Pietro Giannone nell' Istoria civile del Regno di Napoli:

[...] lume maggiore della gloria de' nostri Tribunali, al qual dobbiamo non solo d'aver egli restituita in quelli la vera arte d'orare; ma molto più per avere nel nostro Foro introdotta l'erudizione ed il disputar gli articoli legali secondo i veri principi della Giurisprudenza, e secondo l'interpretazioni de' più eruditi Giureconsulti, de quali presso noi rara era la fama, ed il nome, applicando la lor dottrina all'uso del Foro, ed alle nostre controversie forensi. ${ }^{23}$

Sull'avvio verso la sua carriera forense influì il padre Diego, anch'egli avvocato, ma sicuramente sulla sua vocazione alla cultura umanistica incise lo zio Onofrio, nato nel 1605 e morto con molta probabilità dopo il 1647, poeta e membro dell'Accademia degli Oziosi. Fu autore delle Prose, pubblicate nel 1663 , in cui si discutevano anche i pareri di alcuni filosofi, tra cui Aristotele e Platone; imitò, rielaborandola, la Gerusalemme Liberata di Torquato Tasso con il poema eroico L'ttalia Liberata, inframmezzato da passi in cui riportava le teorie platoniche sull'amore. Nonostante la produzione letteraria di Onofrio non brilli per originalità, sicuramente contribuì alla predisposizione del nipote Francesco verso il nuovo movimento intellettuale che stava attraversando la città. ${ }^{24}$ Anche il fratello Gennaro, nato nel 1637, intraprese l'atti-

22. Ivi, p. 199. Sul rapporto tra Colonna e D'Andrea si veda Nino Cortese, Cultura e politica a Napoli dal Cinque al Settecento, Edizioni Scientifiche Italiane, Napoli, 1965, pp. I3I-I32.

23. Pietro Giannone, Dell'Istoria civile del Regno di Napoli libri XL, per lo Stampatore Niccolò Naso, Napoli, I723, vol. IV, p. 420. L'autore, nella stesura dell'opera, si avvalse di un nutrito gruppo di amici intellettuali, formatisi alla scuola di Gaetano Argento.

24. Si veda Maria Teresa BiagetTi, s.v. «D’Andrea Onofrio», in Dizionario Biografico degli Italiani, Istituto dell'Enciclopedia Italiana, Roma, 1986, vol. 32, ad vocem.

Pedralbes, 4I (2O2I), 255-298, ISSN: O2II-9587, DOI: IO.344/PEDRALBES2O2I.4I.8 
vità forense ed entrò a far parte dell'Accademia degli Investiganti, e a lui si può forse ascrivere la lettera che introduce il testo di Leonardo Di Capua, Lezioni intorno alle mofete, del I683, in cui si mettono in luce le opere di Tommaso Cornelio e di Di Capua come responsabili di aver "portato il primo lume». ${ }^{25}$ I due fratelli, entrambi sostenitori di Cornelio, erano quindi ben consapevoli che gli Investiganti stavano elaborando un nuovo sistema di pensiero, il cui programma, che sancì anche la nascita formale dell'Accademia, si concretizzò nell' Ode in lode della famosa Accademia degli Investiganti, del I665, in cui si sosteneva che la ricerca dei fenomeni naturali andava ricercata attraverso esperimenti da effettuare in assoluta libertà ideologica. ${ }^{26}$ Grande impulso fu dato negli anni dalla regina Cristina di Svezia, che nel 1674 creò a Roma un'accademia in cui furono ospitati proprio due membri investiganti, Giovanni Alfonso Borelli e Luca Antonio Porzio. ${ }^{27} \mathrm{Fu}$ inoltre una let-

25. Ibidem. In un passo significativo dell'opera dedicata ai nipoti, Francesco scrisse di aver appreso da Cornelio «quanto, per assuefar l'ingegno a non ammettere nessuna cosa per vera che non sia dimostrata da suoi principij)(Avvertimenti ai nipoti, p. 229). Sulla traiettoria politica del fratello Gennaro, nominato nel I 686 Fiscale della Sommaria, reggente nel Consiglio d'Italia nel 1689 e reggente del Collaterale nel I695, si veda il capitolo a lui dedicato, in Ivi, pp. 228-235. L'altro fratello era il teatino Gaetano, che intraprese la carriera ecclesiastica e a cui fu concesso il vescovato di Monopoli, nel 1698 (Domenico Confuorto, Giornali di Napoli dal MDCLXXIX al MDCIC, a cura di Nicola Nicolini, Società Napoletana di Storia Patria, Napoli, I930, vol. II, p. 300). Gaetano, lettore e prevosto nella chiesa dei santi Apostoli, svolgeva azione di proselitismo tra i giovani più colti; avrebbe tentato, durante una conversazione in libreria di convincere il giovane Vico a farsi teatino; la notizia dell'aneddoto è fornita da Mastellone, Pensiero politico, p. I42.

26. L'Ode è stata pubblicata da Antonio Borrelli, «Un'ode per l'Accademia degli Investiganti nel nome del "gran Galileo" ", Giornale critico della filosofia italiana, 9I (2012), pp. 19-27.

27. Sul terzo periodo dell'Accademia si veda Fisch, L'Accademia degli Investigan$t i$, pp. 47-55. Cristina di Svezia fondò due volte a Roma una propria accademia: la prima appena arrivata nella capitale, nel 1656 , dalla vita brevissima, e la seconda nel 1674, dal programma più articolato. Sulle accademie fondate a Roma da Cristina, si vedano i diversi contributi nel volume Cristina di Svezia e la cultura delle accademie, in D. Poli, a cura di, atti del convegno internazionale, Macerata-Fermo, 22-23 maggio 
trice appassionata di Leonardo Di Capua, con cui entrò in corrispondenza e che incoraggiò per la pubblicazione delle conferenze tenute nel I664 sui fenomeni dei Campi Flegrei, date poi alle stampe nel I683..$^{28}$

L'Accademia diede inizio ai suoi lavori accogliendo soprattutto discussioni legate ai modelli della rivoluzione europea, come riferito dai due inglesi Philip Skippon e John Ray, membri della Royal Society che si trovavano a Napoli in quegli anni:

At the marquis of Arena's place, 30 June, the Academic Investigants meet every Wednesday in the afternoon, when we observed about 60 persons present. They discourse about several things, and brought in the experiment of water ascending in glas tubuli, or small pipes [...]. They complained to us of the Inquisicion, and their clergymen opposition the new philosophy. ${ }^{29}$

L'Accademia, soppressa nel i668 dal viceré Pedro Antonio de Aragón, proseguì la sua attività, seppur in maniera informale, negli anni che vanno dal I683 al I697, in realtà i più attivi e densi di sviluppi e in cui si registrò la presenza, nelle ultime riunioni, del giovane Giambattista Vico. ${ }^{30} \mathrm{Nel}$ i684 la morte di Tommaso Cornelio sembrò quasi segnare la fine del movimento, ma D’Andrea fece celebrare, nel I685, un solenne

2003, Il Calamo, Roma, 2005. Su Cristina di Svezia come promotrice di cultura e collezionista d'arte, si veda Tomaso Montanari, «Il cardinale Decio Azzolino e le collezioni d'arte di Cristina di Svezia", Studi secenteschi, 38 (1997), pp. I85-264.

28. Sul terzo periodo dell'Accademia si veda Fisch, L'Accademia degli Investigan$t i$, pp. 47-55.

29. Philip Skippon, «An account of a Journey Made Thro'Part of the Low Countries, Germany, Italy, and France», in A. Churchill, ed., A collection of voyages and travels, some now first printed from original manuscripts, Awnsham \& John Churchill, Londra, I732, vol. 6, p. 607. Il passo, che si è preferito trascrivere nella lingua originale, è stato tradotto in italiano da Alberto Labellarte, Atomismo e corpuscolarismo nella Napoli di fine Seicento, Armando Editore, Roma, 2019, p. 28; qui l'autore si rifa tuttavia a un'edizione ancora successiva. In questo saggio, che attraversa le biografie e le opere dei più incisivi membri dell'Accademia degli Investiganti, si offre un quadro molto dettagliato della cultura dell'epoca.

30. Su una panoramica del contesto culturale in cui si formò Vico si veda Harold Samuel Stone, Vico's cultural history. The production and transmission of ideas in Naples, I685-1750, Brill, Leiden, 1997.

Pedralbes, 4I (2O2I), 255-298, ISSN: O2II-9587, DOI: IO.344/PEDRALBES2O2I.4I.8 
funerale in suo onore e scrisse l'Apologia in difesa degli Atomisti, proprio per continuare a riaffermare la vitalità e l'importanza della nuova scienza. I suoi membri continuarono a riunirsi nel salotto di Nicola Caravita, nella libreria di Antonio Bulifon e nella biblioteca di Giuseppe Valletta, luoghi che divennero anche tappe obbligate per tutti coloro che decidevano di visitare Napoli, richiamati dal suo fermento culturale. Fu soprattutto la dimora di Giuseppe Valletta, amico intimo di Francesco D'Andrea, a costituire un punto di riferimento in cui il viaggiatore Burnet scrisse che vi si riuniva un gruppo di intellettuali «mal guardati dal clero, e $[\ldots .$.$] descritti come una setta di ateisti» .^{3 \mathrm{~L}} \mathrm{La}$ sua residenza ospitava più di sedicimila volumi e una variegata collezione d'arte, formata da quadri, medaglie, vasi antichi e statue..$^{32}$ Valletta a partire dagli anni Ottanta abbandonò la sua brillante carriera d'avvocato per dedicarsi agli studi e al sostegno degli Investiganti, ${ }^{33}$ e Francisco de Benavides, IX conte di Santisteban, scelse proprio la dimora di Valletta tra i primi luoghi da visitare non appena arrivato a Napoli: «fra le prime cose, che volle andare vedendo in questa città, poiché con tanto plauso ci giunse, fu la medesima libreria, nella quale buona pezza s'intertenne». ${ }^{34}$

3I. BuRnet, Burnet's Travels, pp. I60-I6I.

32. Sulla sua biblioteca, di respiro europeo, si veda Vittor Ivo Comparato, Giuseppe Valletta: un intellettuale napoletano alla fine del Seicento, Istituto Italiano per gli Studi Storici, Napoli, 1970. In Bernardo De Dominici, Vite de pittori, scultori ed architetti napoletani (1742-1745), ed. a cura di F. Sricchia Santoro e A. Zezza, Paparo Editore, Napoli, 2017, vol. II, p. 888, si parla addirittura di museo: «fu così dilettante di pittura che non solo unì la sua famosa libraria, per la quale si era reso assai chiaro appresso quasi tutti i letterati di Europa, ma a caro prezzo cercò di avere le pitture più rare, anche da oltremontani paesi, per adornare il suo museo». Il contributo più recente su Valletta come collezionista d'arte e sulla tipologia della sua raccolta è di Loredana LoRizzo, «La collezione dell'avvocato Giuseppe Valletta (I636-17I4) tra le carte Orsini», Bollettino d'Arte, 28 (2015), pp. 87-96.

33. Sugli interessi storico-filosofici di Valletta si veda Lucia Giansiracusa, «La giustificazione storica del corpuscolarismo nella Istoria Filosofica di Giuseppe Valletta», Rivista di Storia della Filosofia, I (1988), pp. I8I-19I.

34. Così scrive l'editore Carlo Porpora in una dedica contenuta all'interno della ristampa dell'opera postuma di Pietro Lesena, Dell'antico ginnasio napoletano, a spese di Carlo Porpora, Napoli, I688, pp. [6-7]. 
Benavides era arrivato a Napoli il 26 gennaio 1688 , ma aveva già ricevuto la nomina di viceré di Napoli il 20 dicembre dell'anno precedente, mentre si trovava a Madrid, dove aveva fatto ritorno da pochi mesi dopo l'esperienza di governo in Sicilia (1678-1687). ${ }^{35}$ Ad accoglierlo nella città partenopea furono numerose lettere di enhorabuena, insieme alla paura che potesse mettere in atto la stessa politica repressiva siciliana e al sospetto che avesse comprato la carica vicereale grazie a una consistente somma donata alla regina spagnola. Riuscì invece a dissipare i timori di buona parte della popolazione adottando un comportamento molto cauto nelle spinose questioni lasciate in sospeso dai suoi predecessori e guadagnando così la fiducia di un ampio settore dell'opinione pubblica. La qualificazione di "prudente», che aveva connotato i primi tempi della sua carriera militare, lo accompagnò fino agli anni del suo governo napoletano, quando il Cappellano Diego Vicente de Vidania, incaricato proprio dal viceré Benavides di stendere un memoriale sulle origini e i titoli della famiglia Benavides, enumerando tutti i servicios prestati alla monarchia spagnola, lo presentava come un ottimo e fedele servitore della Corona, dotato di una straordinaria capacità di risolvere con cautela le difficoltà incontrate durante gli anni del go-

35. In una lettera del 3 gennaio I688, conservata nell'Archivo Histórico de la Nobleza di Toledo (AHNo), Osuna, leg. 90-I, f. n.n., Francisco de Benavides comunicò a Gregorio María de Silva y Mendoza, IX duca dell'Infantado e V duca di Pastrana, della sua partenza da Alicante: «quedo ya embarcado para dar principio a mi pasaje a Nápoles en dos naves ingleses». Alla morte di Gaspar Méndez de Haro y Guzmán, VII marchese del Carpio, avvenuta il 6 novembre del I687, era stato nominato come luogotenente a capo della città partenopea, per poche settimane, Lorenzo Onofrio Colonna. Per un affondo sul viceregno napoletano di Benavides, si veda l'imprescindibile capitolo in Galasso, Napoli spagnola, pp. 325-389. Mi permetto di rimandare anche alla mia tesi di dottorato, Paola SETARO, "Principe d'altissimo senno, e di profonda letteratura". La política artística y cultural de Francisco de Benavides, IX conde de Santisteban, virrey en Nápoles (I688-I696)», Universidad Autónoma de Madrid-Università Federico II di Napoli, 202I, parzialmente confluita in EADEM, Ritratto di un viceré. Francisco de Benavides a Napoli tra collezionismo e rappresentazione del potere (I688-1696), Guida, Napoli, 202I.

Pedralbes, 4I (2O2I), 255-298, ISSN: O2II-9587, DOI: IO.344/PEDRALBES2O2I.4I.8 
verno napoletano. ${ }^{36}$ In effetti il viceré si trovò subito a dover fronteggiare problemi di grande portata: la gestione post-terremoto del I688, il provvedimento finanziario del cambio della moneta nel i689, l'emergenza della peste nel i69i e la polemica anticuriale che si stava riaccendendo proprio in quegli anni, anche grazie alle nuove idee teorizzate dagli intellettuali legati all'Accademia degli Investiganti. Sebbene i suoi affiliati dimostrarono fin da subito di non volersi muovere in una dimensione elitaria e di non aver bisogno di sostegno dal mecenate di turno, ma anzi fronteggiarono i poteri ufficiali per dimostrare che la nuova scienza poteva essere applicata alla società, il viceré Francisco de Benavides frequentò assiduamente la dimora di Valletta. ${ }^{37}$ Non si hanno prove di un suo diretto coinvolgimento nelle riunioni degli accademici, ma è significativo che nella biblioteca di Benavides una piccola sezione fosse costituita da alcuni trattati di filosofia morale, come quelli di Seneca, Tacito, Giusto Lipsio. ${ }^{38}$ Pur non volendo addentrarsi nell'analisi delle implicazioni intellettuali, è significativo evidenziare che l'avvicinamento alla corrente dello stoicismo, esponente di quella saggezza antica che si allontanava dagli assunti principali della cultura cristiana, costituì una delle principali componenti del rinnovamento culturale che stava animando non solo Napoli ma molte altre città italiane. Furono proprio le biblioteche, i salotti e le accademie a diventare i luoghi privilegiati per la diffusione di quelle

36. Diego Vicente de Vidania, Al Rey Nuestro Señor, Don Francisco de Benavides Dávila Corella y de la Cueva, IV del nombre, IX conde de Santisteban del Puerto, representa los servicios heredados y propios y los de su hijos [...], Parrino e Mutii, Napoli, I696, pp. 408-439.

37. Si legge nella pagina degli Avvisi del 25 maggio I694, conservati in Biblioteca della Società Napoletana di Storia Patria, Napoli (BNSP), n. 32: «[...] nella scorsa domenica la spinse a portarsi di nuovo nella celebre e famosa libreria dell'eruditissimo Avvocato Sig. Giuseppe Valletta, dove è più di una volta stata l'Eccellenza Sua quale si trattenne ancora nell'appartamento e galleria».

38. Si veda Luis Riвot, «El IX Conde de Santisteban (I645-1716). Poder y ascenso de una casa noble a través del servicio a la Corona», Espacio, Tiempo y Forma - Serie IV. Historia moderna, 3I (2018), p. 29. 
idee a orientamento libertino che stavano iniziando a mettere in discussione i principi dell'autorità della Chiesa. ${ }^{39}$

Anche sul versante scientifico si comincia a dare spazio a interessi poco ortodossi, tra cui il "curioso», che comincia a diventare oggetto di studio e poi di collezioni. A Napoli spicca su tutte la residenza di Francesco Antonio Picchiatti, ingegnere e architetto napoletano che ospitava un «museo d'anticaglie curiose», anche questo conosciuto dal viceré Benavides, come ci riportano le fonti del tempo..$^{40} \mathrm{Il}$ rapporto che legò Benavides agli intellettuali napoletani dovette quindi essere molto stretto, se anche dalle parole di Carlo Ruzzini, ambasciatore veneziano alla corte di Spagna, trapela il suo sostegno a quella corrente della filosofia moderna che stava prendendo piede proprio negli anni del suo governo:

La controversia per l'Inquisizione di Napoli arde più che mai non solo fra le contese ma quasi fra i tumulti; fan conoscere quei popoli, como lo ritiene quel Viceré, la più animata risolutione per non tolerar alcun immagine, et autorità di quell'ufficio; anzi per interamente scioglierla mandano al Re che le cose ritornino alla purità; et unione dei suoi principii. ${ }^{41}$

È il 3I luglio del I692 e Napoli, nel resoconto del veneziano, viene descritta come un universo in fermento che sta resistendo strenuamente agli abusi perpetrati dal Tribunale del Sant'Ufficio e che trova nel

39. In questo panorama un ruolo centrale fu rivestito da Roma, che emerge come un teatro barocco capace di attirare numerose personalità interessate al nuovo rapporto tra uomo e natura; a tal proposito si veda il testo di Sabina Brevaglieri, Natural desiderio di sapere. Roma barocca tra vecchi e nuovi mondi, Viella, Roma, 2009. Sul fenomeno del libertinismo, che investì inevitabilmente anche il campo delle arti figurative, si veda Dalma Frascarelli, L'arte del dissenso. Pittura e libertinismi nell'Italia del Seicento, Torino, Einaudi, 2016.

40. CONFuORTO, Giornali, I, p. 216. Sulla wunderkammer di Picchiatti si rimanda al contributo di Luigi Abetti, in cui viene trascritto anche il suo inventario dei beni finora inedito: Luigi AвEтTI, «Artificialia e naturalia nella casa-studio di Francesco Antonio Picchiatti», Ricerche sull'arte a Napoli in età moderna, 2016, pp. 52-77.

4I. Archivio di Stato di Venezia (ASVe), Senato. Dispacci degli Ambasciatori. Spagna, Filza I29, nn. 3I-32, 3I luglio I692, p. n.n.

Pedralbes, 4I (2O2I), 255-298, ISSN: O2II-9587, DOI: IO.344/PEDRALBES2O2I.4I.8 
viceré un alleato, interessato a difendere quei privilegi ottenuti già dai primi anni del viceregno e grazie ai quali si era riusciti finora a tenere lontano «l'obbrobrio della Inquisizione». ${ }^{42}$

\section{La polemica anticuriale e il "processo agli ateisti»}

È quindi proprio al ruolo degli Investiganti che va attribuito il merito di quel rinnovamento orientato verso una cultura libera dal pregiudizio religioso. Se dal punto di vista filosofico aspiravano a una libertà di stampo cartesiano, da quello politico e giuridico ciò che rivendicavano era la libertà di azione nel diritto privato, in uno Stato in cui i poteri dovevano essere regolati dalla legge..$^{43}$

L'obiettivo era soprattutto contrastare l'operato del Tribunale del Sant'Uffizio, istituito da Paolo III nel I542, che si serviva, per realizzare la sua azione di controllo della cultura e repressione delle eresie, di ministri delegati e poi di vescovi. ${ }^{44}$ Falliti i tentativi di instaurare l'Inquisizione spagnola, a Napoli si creò un sistema particolare e complesso: in materia inquistoriale erano i vescovi ad agire ma con il sostegno

42. L'espressione è tratta dall'iscrizione sulla lapide che si trova nella napoletana Certosa di San Martino, datata I547, che ricorda le sommosse del popolo napoletano all'introduzione, da parte del viceré Pedro de Toledo, del tribunale romano in città: «Ai popolani di Napoli che nelle tre oneste giornate del luglio MDXLVII, laceri, male armati e soli d'Italia francamente pugnando nelle vie, dalle case contro le migliori armate d'Europa tennero da sé lontano l'obbrobrio della Inquisizione Spagnola imposta da un imperatore fiammingo e da un papa italiano e provarono anche una volta che il servaggio è male volontario di popolo ed è colpa dei servi più che de padroni».

43. Si veda Maurizio Torrini, «Descartes e il cartesianesimo nelle corrispondenze italiane al tempo della rivoluzione scientifica», Rivista di Filosofia Neo-Scolastica, 93 (200I), n. 4, pp. 550-570.

44. Una recente chiave di lettura non considera l'iniziativa inquisitoriale un mero strumento di cui si servivano i pontefici per portare avanti l'impegno antiereticale, ma un obiettivo per realizzare un programma dai risvolti prevalentemente politici. Un bilancio sullo stato della ricerca è in Michaela VALEnte, "Nuove ricerche e intepretazioni sul Sant'Uffizio a più di dieci anni dall'apertura dell'archivio", Rivista di Storia della Chiesa in italia, 2 (2012), pp. 569-592. 
degli inquisitori nominati da Roma. ${ }^{45}$ La lotta all'eresia era quindi portata avanti dagli ordinari tribunali vescovili in cui tuttavia operava, dietro le quinte, un commissario del Sant'Uffizio. Le politiche repressive messe in atto dalla Curia napoletana nella seconda metà del xvir secolo erano incentrate soprattutto sulla repressione dei comportamenti non ortodossi sotto il profilo religioso, ma anche su quegli aspetti della vita quotidiana che andavano dalle pratiche magiche e sortilegi ad argomenti della morale familiare e privata. ${ }^{46} \mathrm{Tra}$ i punti fondamentali alla base della reazione anticuriale c'erano anche la censura dei libri e le immunità della Chiesa, questioni che anche il governo spagnolo cominciò a sentire vicine, perché interessato a non soccombere alle autorità ecclesiastiche. Quello che si metteva in discussione era quindi la legittimità dell'uso della giustizia da parte della Chiesa. Lo spunto per la polemica era nato in seguito alla pubblicazione, nel I677, della Risposta al Trattato della Regina cristianissima sopra il ducato di Brabante e altri Stati della Fiandra, opera di Francesco D'Andrea, il quale metteva in luce per la prima volta l'importanza del diritto naturale su quello privato. ${ }^{47} \mathrm{Il}$ contributo argomentava intorno alla problematica questione nata in segui-

45. Sull'argomento la bibliografia è molto vasta. Oltre ai testi, ancora attualissimi, di Luigi Amabile, Il Santo Officio della Inquisizione in Napoli, Lapi, Città di Castello, I892, in particolare le pp. 54-75, in cui si offre una panoramica sulle diverse forme attraverso cui il tribunale agiva, e Giovanni Romeo, «Per la Storia del sant'Ufficio a Napoli tra '5oo e '6oo. Documenti e problemi», Campania Sacra, VII (I976), pp. 5-109, in cui si mette a fuoco, attraverso la pubblicazione degli atti di alcuni processi, l'efficacia del suo funzionamento chiaramente dipendente da un complesso ventaglio di situazioni, si veda anche il lavoro di Vittoria Fiorelli, I sentieri dell'inquisitore. Sant'Uffizio, periferie ecclesiastiche e disciplinamento devozionale (I6I5-1678), Guida, Napoli, 2009.

46. Sui modelli di controllo delle coscienze, si veda Adriano Prosperi, Tribunali della coscienza, Inquisitori, confessori, missionari, Einaudi, Torino, I996, p. 74.

47. Sulla dimostrazione dell'ingiustizia di tali pretese, si veda Salvo Mastellone, Francesco d'Andrea politico e giurista (I648-1698). L'ascesa del ceto civile, Olschki, Firenze, I969, pp. I5-50. D’Andrea, sostenitore dell'urgenza di applicare le nuove idee agli studi giuridici, aveva fatto assegnare le cattedre dello Studio di Napoli a quegli intellettuali che avrebbero potuto garantire una diffusione capillare della nuova cultura, cioè Domenico Aulisio, Tommaso Cornelio, Leonardo Di Capua e Gregorio Messere.

Pedralbes, 4I (2O2I), 255-298, ISSN: O2II-9587, DOI: IO.344/PEDRALBES2O2I.4I.8 
to alla guerra di Devoluzione (I667-I668), esplosa dopo la morte di Filippo IV, nel I665, quando Luigi XIV rivendicò per la moglie Maria Teresa, figlia di primo letto del re defunto, una parte dei suoi domini, poiché non era stata pagata l'enorme dote pattuita per la rinuncia di lei all'eredità. I domini erano quelli in cui non era in vigore la legge salica che escludeva le donne dalla successione; secondo questo diritto, l'eredità spettava solo ai figli di primo letto e non a quelli di secondo, quale era il re Carlo II..$^{8}$ Per D’Andrea il diritto accampato dal re francese rientrava nella sfera di quello privato, quindi non applicabile alle successioni reali, regolate al contrario dal diritto pubblico.

Contemporaneamente a questo dibattito che si faceva sempre più vivo, anche la crisi economica e sociale di Napoli stava raggiungendo un limite pericoloso, poiché i baroni stavano cominciando a esercitare pressioni per ricoprire cariche nel governo cittadino, mentre la monarchia spagnola, che fino ad allora aveva concesso alla feudalità il diritto di nominare funzionari amministrativi e giudici, trovò un alleato proprio nel ceto civile, che aspirava ad acquistare titoli e a occupare una posizione sempre più rilevante nel governo. ${ }^{49}$

Accanto a Francesco D'Andrea, anche l'amico Giuseppe Valletta partecipò attivamente alla lotta antifeudale e anticuriale. Condividevano non solo l'idea che il governo vicereale avesse concesso troppi diritti ai baroni, arrivando a far usurpare quelli regi, tra cui l'imposizione dei dazi, ma soprattutto quella sulle ingiustizie commesse dal Tribunale dell'Inquisizione. Con la memoria sulla questione della sua introduzione a Napoli, Al nostro SS.mo Padre Innocenzo XII, Intorno al Procedi-

48. I due, che erano due volte cugini primi, si sposarono nel I660, l'anno successivo al trattato dei Pirenei, che sancì i confini tra Francia e Spagna. Fu in quest'occasione infatti che fu stipulato il matrimonio: Luigi xIV accettò di sposare Maria Teresa dietro la promessa da parte di Filippo IV di 500.000 scudi come contropartita della rinuncia di quest'ultima a qualunque parte dell'eredità spagnola. Sulla guerra di Devoluzione, si veda Antonio José Rodríguez Hernández, España, Flandes y la Guerra de Devolución, Ministerio de Defensa, Madrid, 2007.

49. Sul riconoscimento del ceto forense da parte del governo vicereale, si veda Raffaele Colapietra, Vita pubblica e classi politiche nel viceregno napoletano (I6s61734), Edizioni di Storia e Letteratura, Roma, I96I. 
mento ordinario e canonico nelle cause che si trattano nel Tribunale del Sant'Uffizio nella città e Regno di Napoli, redatta nel i693 e poi rielaborata negli anni successivi, Valletta si inseriva in un più ampio dibattito di respiro europeo sull'Inquisizione, ripercorrendo la traiettoria della Chiesa, dalle origini ai tempi attuali. In particolar modo pose in evidenza l'arretratezza del Tribunale dell'Inquisizione, regolato da leggi contro natura:

[...] che l'origine di questi nuovi rigori dell'Inquisizione ella è stata a causa, che vedendosi pur troppo fuora de' Chiostri dilalate le lettere e propagata nella nostra Patria la Filosofia; [...] si son lasciati a dire, che quella fosse di pregiudizio alla nostra fede. ${ }^{50}$

Con uno scritto di poco successivo, del I695-96, il Discorso filosofico in materia d'Inquisizione e intorno al corregimento della Filosofia di Aristotele, Valletta arriva a una consapevolezza più matura della lotta all'Inquisizione, che secondo il giurista doveva certamente essere portata avanti contrastando il potere con un richiamo giurisdizionale, ma anche ricondotta alla dimensione ideale della nuova cultura. ${ }^{5 \mathrm{I}}$

Contro le nuove idee era già stato imbastito qualche anno prima un processo a opera del Tribunale delegato dell'Inquisizione, che segnò la reazione della Curia romana e napoletana alla diffusione dell'atomismo e del pensiero di Gassendi e Cartesio. La libertas philosophandi, stimolando una verifica della credibilità della fede tradizionale, costituiva una seria minaccia per la stabilità politica ecclesiastica. Un chiaro segno fu che nel processo non furono accusati solo quelli che salirono sul banco degli imputati, ma tutti gli intellettuali coinvolti nella nuova corrente culturale.

50. Questo inserto filosofico è presente in tutte le stesure della lettera a Innocenzo XII, conservate, in forma manoscritta, nella Biblioteca Nazionale di Napoli e nella BSNSP(nella sua versione latina); la notizia è fornita da Comparato, Giuseppe Valletta, 1970, p. 195.

51. Sugli elementi di novità della riflessione civile di Valletta, vedi Biagio De Giovanni, "Cultura e vita civile in Giuseppe Valletta», Studi sul Settecento Italiano, Istituto Italiano per gli studi storici, Napoli, 1967, pp. I-47. 
La polemica si scagliò soprattutto contro le competenze e i metodi adottati dal tribunale del Sant'Uffizio a Napoli; $;^{2}$ fu quindi per il tentativo di fermare la diffusione delle nuove idee che quest'ultimo imbastì quello che passerà alla storia come il "processo agli ateisti», iniziato nel I688 e protrattosi fino al I697. A questo punto è doveroso ricordare che non fu frutto di una reazione improvvisa di Roma, ma di un episodio accaduto nel I67I, quando la Congregazione dell'Inquisizione aveva comunicato per iscritto al cardinale Innico Caracciolo, allora arcivescovo di Napoli, che alcuni intellettuali stavano applicando le idee di Cartesio al campo teologico, incitandolo a comunicare alla stessa Congregazione la pericolosa nascita di quelle opinioni. ${ }^{53}$ I principali accusati furono due avvocati molto stimati nell'ambiente culturale napoletano, Basilio Giannelli, ricordato anche per la sua attività poetica, e Giacinto De Cristoforo, matematico e autore di due ponderose opere, una dedicata alle biografie degli accademici pontaniani e l'altra a una storia delle scienze e delle lettere, oltre a due personaggi che si trovavano a Napoli per terminare i loro studi di legge, Filippo Belli e Francesco Paolo Manuzzi, quest'ultimo giovane avvocato barese, i quali alla fine del processo abbandonarono la città senza lasciare alcuna traccia. ${ }^{54}$

52. Sull'anomalia del Sant'Ufficio a Napoli in età moderna e sulle ribellioni intraprese dagli organi di governo cittadino contro la paventata immissione di una sede del tribunale spagnolo e romano, le cui trattazioni non possono trovare spazio in questa sede, si rimanda ad alcuni contributi, innanzitutto quelli tradizionali di Giovanni Romeo, «Una città, due Inquisizioni: l'anomalia del Sant'Ufficio a Napoli nel tardo '500", Rivista di storia e letteratura religiosa, 24 (1988), pp. 42-67; IDEM, L'Inquisizione nell'Italia moderna, Laterza, Roma-Bari, 2002, pp. I2I-I33. Si vedano inoltre il saggio di Elisa Novi ChavarRia, «Procedure inquisitoriali e potere politico a Napoli (I550-I640)", in I primi Lincei e il sant'Uffizio: questioni di scienza e fede, atti del Convegno dell'Accademia Nazionale dei Lincei, Roma I2-I3 giugno 2003, Roma, Bardi, 2005, pp. 3I-46, e quello di Mario DEL TREPPO, «Il sant'Ufficio dell'Inquisizione a Napoli», Napoli nobilissima, v serie, 3-4 (7/2007), pp. 152-158.

53. Si veda Luciano Osbat, L'Inquisizione a Napoli. Il processo agli ateisti (I6881697), Edizioni di Storia e Letteratura, Roma, 1974, in cui la vicenda viene intepretata dallo studioso soprattutto come strumentalizzazione politica da parte della Chiesa.

54. Si vedano Giuseppe Galasso, «Napoli nel viceregno spagnolo (I696-1707)», in Storia di Napoli. Fra Spagna e Austria, Società Editrice Storia di Napoli, Napoli, 
Questo episodio scosse profondamente la città, segnando l'inizio di un periodo di reazione da parte della Curia romana e di quella napoletana nei confronti delle nuove tendenze culturali, tendenze che avevano conquistato molto rapidamente non solo laici, ma anche componenti del clero. Le conseguenze del processo produssero inoltre una profonda frattura anche all'interno del mondo intellettuale, che si rese evidente negli anni Novanta del XVII secolo.

La Curia romana, fin dall'inizio, si era posta come obiettivo principale quello di stroncare la diffusione della filosofia moderna, per ristabilire la solidità della religione cristiana; è stato tuttavia ipotizzato che in realtà la vicenda del processo fosse stata pianificata dai gesuiti, per il timore che le nuove scuole potessero in qualche modo turbare il monopolio della cultura da loro gestita. Al di là dell'interpretazione della storiografia di questo momento storico determinante, come reagì nei fatti il ceto civile e come gestì la situazione il viceré Francisco de Benavides? Per rispondere a queste domande si devono necessariamente fare alcuni passi indietro nella ricostruzione delle vicende, partendo dal 2I marzo I688, quando Manuzzi si presentò a Giuseppe Nicola Giberti, vescovo di Teano e allora delegato del Sant'Uffizio nel viceregno, per denunciare la presenza a Napoli di Basilio Giannelli e Giacinto De Cristofaro.

La sentenza non provò davvero se le colpe fossero state effettivamente commesse, ma a ogni modo terminò con pene severe, comminate da Giovanni Battista Giberti, vescovo di Cava, che aveva sostituito il suo predecessore nella carica di ministro delegato del Sant'Uffizio: Giannelli rimase in carcere tre mesi e fu costretto a restare lontano da Napoli per alcuni anni e De Cristofaro scontò sei anni di carcere.

L'inquisitore Giberti, oltre ai processi contro Giannelli e De Cristofaro, aveva inoltre dimostrato la volontà di incarcerare anche alcuni funzionari dell'amministrazione vicereale, tra cui l'avvocato Francesco D’Andrea, ma il viceré si oppose fermamente, non concedendo l'auto-

1970, vol. 6 (Tra Spagna e Austria), pp. 55-85, e Vittoria Fiorelli, «Ateisti», in A. Prosperi, J. Tedeschi, a cura di, Dizionario storico dell'Inquisizione, Edizioni della Normale, Pisa, 2oro, vol. I, ad vocem. 
rizzazione e giustificandola con il motivo "di non poter carcerare ministri senza l'ordine di sua Maestà, e che perciò ne scrivesse alla corte».55 La reazione di Benavides destò molto scalpore agli occhi delle Piazze e non fu giudicata in una maniera del tutto positiva da alcune fonti contemporanee, che lo accusarono di prepotenza per aver messo in subbuglio i poteri locali. ${ }^{56}$ Eppure si configura come una spia significativa della linea politica attuata dal "teorico dai tempi relativamente lunghi», ${ }^{57}$ che nei limiti del suo esercizio del potere, difese e sostenne la polemica anticuriale, andando «in concerto" con la città di Napoli..$^{58}$ A differenza di modalità che nel passato avevano provocato tumulti popolari, Benavides avrebbe invece favorito una trattativa durata due anni, dal I693 al I695, tra l'ambasceria napoletana e i cardinali deputati da Innocenzo XII a trattare con questa. Non era propenso agli scontri frontali, anche perché la sua preoccupazione principale era quella di evitare dissidi con le volontà di Carlo II, che nel I694 gli conferì la prestigiosa nomina di Grande di Spagna, titolo a cui Benavides aveva sempre aspirato. ${ }^{59}$ In questo senso cercò sempre un dialogo anche con il cardinale Cantelmo, che nell'estate del I69I fu designato dal pontefice come nuovo delegato del Sant'Uffizio a Napoli: anche quest'ultimo riteneva fondamentale agire con diplomazia e cautela nei confronti dell'aristocrazia e della borghesia più influente. ${ }^{60}$ Tuttavia Sebastián de Cotes y Cárcel, testimone della tormentata vicenda in quanto in quegli anni presidente della Sommaria, metteva in evidenza, in una lettera

55. Confuorto, Giornali, I, p. 359. La notizia dell'aneddoto è in Giuseppe CoNiglio, I viceré di Napoli, Fausto Fiorentino, Napoli, 1967, pp. 335-336.

56. Ibidem.

57. La definizione è di Osbat, L'Inquisizione a Napoli, p. 222.

58. Confuorto, Giornali, in, p. 4.

59. Il documento di nomina, conservato in Archivo Ducal de Medinaceli, Toledo (ADM-To), leg. 290, doc. I2, è citato per la prima volta da Giulia FusconI, «Il "buen gusto romano" dei viceré. La ricezione dell'effimero barocco a Napoli negli anni del Marchese del Carpio (I683-I687) e del Conte di Santisteban (I688-I696)», in Le dessin napolitain, atti del Colloquio internazionale, Parigi, École normale supérieure, 6-8 marzo 2008, De Luca, Roma, 2010, p. 220, nota 34.

6o. Оsват, L'Inquisizione a Napoli, p. 223. 
destinata all'Almirante di Castiglia, che nonostante il governo del viceré «no puede ser mejor ni en el zelo ni en la providencia con que dispone todo", era anche naturale che "padece necesariamente las debilidades de la edad en muchas dispocisiones». ${ }^{61}$ Nella missiva, scritta da Madrid nel I695, anno in cui ricopriva l'incarico di reggente del Consiglio d'Italia, de Cotes y Cárcel esplicita di essere stato incaricato da Benavides di mettere per iscritto qualche riflessione sul governo; si affronta in esse proprio il problema dell'Inquisizione, sottolineando che per la sua soluzione «necesitaría de un virrey nuevo, y que se reciviese con pretensiones de muy larga vida, porque siendo preciso que este negocio tan critico y peligroso". ${ }^{62}$

La percezione era quindi, da parte di un uomo di Stato che aveva vissuto dall'interno la vicenda, quella di un governo stanco, che aveva già dovuto affrontare molti pericoli interni che lo avevano inevitabilmente indebolito. Nonostante questo, Benavides gestì i disordini con grande energia, come si evince dai racconti di Tiberio Carafa nelle sue Memorie. ${ }^{63} \mathrm{Nel}$ testo, che ripercorre l'intera vicenda, si sottolinea infatti che in questo momento così delicato la preoccupazione principale di Benavides, anche qui definito «uomo accorto e prudente», fu quella di non fomentare disordini ma anzi di assicurare la difesa della città «nell'affare del sant'Officiom. ${ }^{64}$

L'inizio dei disordini si fa risalire all'arrivo dell'arcivescovo Giacomo Cantelmo, il quale non fece altro che rendere ancora più frontale lo scontro tra la Chiesa e coloro che si ribellavano ai suoi soprusi. Diede corso alla denuncia del I688, riaprendo, con l'arresto dei tre accusa-

6I. Fundacíon Bartolomé March, Palma di Maiorca (FBM), Copia de papel que Sebastian de Cotes escribiò al Almirante de orden de Su Excelencia, B82-F-05, 28 agosto I695, f. 26.

62. Ibidem.

63. Memorie di Tiberio Carafa principe di Chiusano, voll. 3, riproduzione in fac simile a cura di A. Pizzo, Società napoletana di Storia Patria, Napoli, 2005. Carafa, nella disamina delle motivazioni che spingevano i napoletani ad opporsi al Tribunale dell'Inquisizione, incentrava il discorso, come aveva già fatto Valletta, sull'iniquità delle procedure speciali utilizzate dai vescovi.

64. Ivi, tomo I, [p. 96], 
ti, la questione della legittimità del Sant'Uffizio nella città partenopea ma sottovalutando la reazione violenta e unanime dei seggi nobili e popolari, da sempre temuta dal governo spagnolo per il suo difficile controllo, e il rapporto ormai molto tenace stabilito tra nobiltà, potere vicereale e nuova corrente filosofico-scientifica. ${ }^{65}$ I seggi, secondo il racconto di Carafa, convocarono una riunione in San Lorenzo il I4 settembre del I69I, durante la quale si stese una relazione in cui si criticavano con durezza le responsabilità dei vertici ecclesiastici nelle tensioni che avevano attraversato la città e si affrontava il rapporto con il governo vicereale. La plebe cominciò però a ribellarsi e i deputati cercarono di sedare il tumulto, assegnando ad Antonio Plastena, eletto del Popolo, il compito di impedire nuove carcerazioni e di consegnare la memoria al viceré. ${ }^{66}$ I rappresentanti del Seggio di Nido volevano inoltre che fosse rispettata, nell'azione contro il Tribunale dell'Inquisizione, una procedura già adottata in un caso analogo nel I66I e che si era risolta con l'espulsione di monsignor Camillo Piazza, in quegli anni inquisitore delegato a Napoli. ${ }^{67}$ Il giorno successivo Benavides, preoccupato

65. I nobili avevano diritto a partecipare a cinque di questi seggi, mentre il resto dei cittadini confluiva in un sesto seggio, quello del Popolo. Sulle pratiche politiche di questa componente municipale di Napoli in età moderna e sulla sua dialettica con il governo vicereale, si veda Piero Ventura, «La capitale e le élites urbane nel regno di Napoli tra XVI e XviII secolo", Mélanges de l'École Française de Rome. Italie et Méditerranée, I (2009), pp. 26I-296, http://mefrim.revues.org/I207 [ultimo accesso I8/04/202I].

66. Queste vicende sono oggetto della documentazione conservata presso l'Archivio Storico Municipale di Napoli, andata distrutta in un devastante incendio nel 1946; resta traccia delle descrizioni in Catalogo ragionato dei libri, registri e scritture esistenti nella sezione antica o prima serie dell'archivio municipale di Napoli (1387-I806): parte I $e$ II, a cura di B. Capasso, ristampa parzialmente riveduta e corretta dell'edizione del I899, 2 voll., Laveglia e Carlone, Battipaglia, 20II, e Catalogo ragionato dei libri, registri e scritture esistenti nella sezione antica o prima serie dell'archivio Municipale di Napoli (1387-I806): parte III, a cura di R. Parisi, Giannini, Napoli, I920.

67. Il fulcro intorno a cui si sviluppò la rivolta fu la confisca dei beni come effetto del rito inquisitorio. In questo specifico episodio il vescovo Piazza appoggiò il decreto di confisca dei beni promulgato dal Consiglio Collaterale l'8 gennaio I66I nei confronti dell'ebreo Odoardo Vaaz, conte di Mola; per minacciare la nobiltà napole- 
dalla prospettiva di un'alleanza tra nobili e ceto civile, ma interessato a salvaguardare le posizioni di entrambe le parti, ordinò di impedire nuove carcerazioni e assicurò di agire da quel momento in poi solo a favore della città. ${ }^{68}$

Nel frattempo i rapporti tra autorità civili ed ecclesiastiche peggioravano sempre di più e un anno dopo il pericolo di una nuova agitazione, nato dalla volontà del seggi di allontanare Cantelmo, spinse Benavides a chiedere il parere del Collaterale e del ceto togato, riconoscendo nel primo interlocutore un ruolo decisivo della volontà politica del governo, e nel secondo una più attiva responsabilità civile. Il viceré cercò di convincere l'arcivescovo a consegnare alla città gli inquisiti che si trovavano nel suo carcere ma Cantelmo rispose che solo il pontefice avrebbe potuto prendere decisioni in merito. Come conseguenza la Curia romana affidò a Cantelmo unicamente poteri commissariali, lasciando intravedere, in quest'atteggiamento moderato, uno spiraglio di luce. Ma si trattò di un compromesso apparente. Nell'ottobre del I692 Cantelmo continuò ad agire con i suoi soprusi, disponendo un'ispezione dei maestri di scuola, a cui il potere regio si oppose, e l'arresto di tutti quelli che erano sfuggiti all'azione di Giberti. ${ }^{69}$ Non era certamente la prima volta che un arcivescovo, per imporre la propria giurisdizio-

tana fece anche arrestare due servi del conte delle Noci, momento in cui la nobiltà cominciò a temere realmente un ulteriore indebolimento del suo potere politico. Fu quella l'occasione in cui si costituì la Deputazione contro il Sant'Uffizio; si procedette all'elezione di sei deputati. Ogni piazza nobile elesse tre rappresentanti, mentre quella del Popolo ne elesse sei: Pietro Di Fusco, Giacinto de' Mari, Giuseppe Costantino, Serafino Biscardi, Francesco Sernicola e Antonio Pistone. Sulle vicende si rimanda a Maria Sirago, "L'Inquisizione a Napoli nel I66I», Quaderni dell'istituto di scienze storico-politiche della facoltà del magistero dell'università di Bari, 1980, pp. 429-454; Claudia Möller Redondo, «El pronóstico de las cruces: intereses políticos e imagen napolitana de la Inquisición "a la manera española" en torno a los sucesos de I66I», in Sardegna, Spagna, Mediterraneo, Atlantico dai Re Cattolici al Secolo d'Oro, a cura di B. Anatra, G. Murgia, Carocci, Roma, 2004, pp. 445-464.

68. Un resoconto dettagliato delle vicende è offerto da Galasso, Napoli spagnola, pp. 442-473, a cui si fa riferimento anche nelle pagine successive.

69. Ivi, pp. $454-458$. 
ne ecclesiastica, entrasse in conflitto con l'aristocrazia ma soprattutto con l'autorità viceregia. Un precedente emblematico si rintraccia in Ascanio Filomarino e nelle tensioni, che in quel caso arrivarono a creare una vera e propria spaccatura, con il viceré Ońate, nel I648.70 Anche nel caso di Benavides, nonostante l'opinione di Carafa sul viceré «sedotto» dal cardinale e arcivescovo Cantelmo, si crearono forti tensioni, che tuttavia non raggiunsero mai livelli preoccupanti. L'arcivescovo, per nulla intimorito, continuò comunque ad agire con i suoi soprusi contro quelle che riteneva eresie. ${ }^{\text {I }}$ Nel mese di settembre del I692 si tenne finalmente l'interrogatorio di Giacinto de Cristofaro che, nel quadro della lucida percezione del pericolo costituito dal nuovo clima culturale dimostrata dall'arcivescovo, segnò la ripresa del processo, affidato al gesuita Domenico Jameo. Intanto la complicità che si era creata tra la Piazza del Popolo e le Piazze dei Nobili per procedere all'espulsione del Cantelmo, mise in allarme Benavides, il quale vietò di riunirsi, riproponendo così quel potere discrezionale del viceré che ledeva alla radice l'autonomia cittadina e le possibilità di azione legale dell'aristocrazia. A questo punto i Deputati si riunirono con i governatori delle Piazze per presentare ricorso a Benavides, che non li ricevette ma che giustificò, con la sua consueta prudenza, la sua posizione. Si difese innanzitutto dall'accusa di connivenza con il Cantelmo, proponendo di rimettere la questione direttamente al Papa. Il pontefice allontanò così Cantelmo, convinto dalle "preghiere del signor viceré di togliere cotal tribunale da questa città e Regno» $»^{72}$ e Benavides potè ordinare l'abolizione delle carceri del Sant'Uffizio, presenti in locali appositi della Curia vescovile.

70. Su quella che Giuseppe Mrozek Eliszynsky ha definito una «guerra di scritture», si veda il suo lavoro su Ascanio Filomarino: Giuseppe Mrozer Eliszynsky, Ascanio Filomarino. Napoli, Chiesa e potere nell'Italia del Seicento, Viella, Roma, 2017, in particolare pp. 169-205.

7I. In Colapietra, Vita pubblica, si attribuisce un ruolo decisivo a Cantelmo come responsabile delle repressioni.

72. Confuorto, Giornali, I, p. 365. 
Si decise inoltre di inviare a Roma due deputati per presentare istanza al pontefice Innocenzo XII: Pietro Di Fusco e il marchese di Pontelatrone. Quest'ultimo, non potendo affrontare il viaggio, fu sostituito da Mario Loffredo, marchese di Monteforte, "soggetto assai degno e per qualità, per erudizione e per buoni costumi $\gg^{73}$ che si mostrò particolarmente ostinato e radicale nelle posizioni esposte dalla Deputazione del Sant'Ufficio contro la Curia romana. Giunto con quest'ultimo a Roma, Pietro Di Fusco, rappresentante del seggio del Popolo, presentò un'orazione al Papa. Tuttavia la questione non fu risolta e le vessazioni non terminarono, anche se nel corso del 1693 vennero ascoltati numerosi testimoni senza che però il Tribunale riuscisse mai a individuare precise e chiare responsabilità ereticali nell'accusato: dalle deposizioni infatti emergeva sempre un quadro caratterizzato da indizi generici.

L'autore delle Memorie riporta inoltre che fu grazie al nipote di Benavides, Luis de la Cerda y Aragón, IX duca di Medinaceli, in quegli anni ambasciatore di Spagna a Roma e prossimo ad assumere l'incarico di viceré di Napoli, e alle sue pressioni su Di Fusco, che la situazione sembrò che stesse per concludersi diplomaticamente; ${ }^{74}$ il duca di Medinaceli riuscì infatti a convincere il ceto civile ad accettare una formula di compromesso sulle vicende del tribunale del Sant'Ufficio. Tuttavia neppure l'intervento dell'ambasceria romana ottenne un risultato definitivo, poiché le proteste ripresero nell'agosto del 1695 , fino a una serie di episodi di forte conflitto che si verificarono nell'estate del I699; ma la presenza a Napoli del nuovo viceré duca di Medinaceli (I695-I702) responsabile della rottura del sodalizio tra ceto civile e nobiltà, fino a quel momento contro i soprusi ecclesiastici, aveva contribuito a stemperare definitivamente la tensione anti-inquisitoriale. ${ }^{75}$ I due gruppi, in realtà, erano stati spinti ad agire nella stessa direzione più dall'affanno della scalata sociale che

73. Ivi, II, p. 83.

74. Osbat, L'Inquisizione a Napoli, p. 28.

75. Ivi, p. 38. 
dall'affermazione di una nuova cultura; ${ }^{76}$ il loro allontanamento si rese evidente proprio quando non ci fu più il pericolo che l'iniziativa della battaglia contro la Curia romana divenisse prerogativa dell'uno o dell'altro. ${ }^{77}$

Gli eventi turbolenti che avevano fortemente condizionato la vita napoletana furono quindi affrontati da Benavides e dal suo successore con grande sensibilità e attenzione verso la cultura moderna, ma fu soprattutto il primo a difendere l'autonomia del libero pensiero dalle prevaricazioni delle istituzioni ecclesiastiche. Lasciò Napoli nel I696, e proprio alla vigilia della sua partenza chiese al filosofo Giambattista Vico un "attestato pubblico ${ }^{78}$ che mettesse in luce la sua politica di sostegno alle spinte autonomistiche del ceto civile. Vico, con dovuto e retorico ossequio ma credendo nella sua apertura intellettuale, gli dedicò un'orazione, che fu inserita come introduzione alla raccolta di componimenti poetici dedicati a Benavides dal giurista Nicola Caravita, ${ }^{79}$ come racconta lo stesso Vico nella Vita di se medesimo:

76. Sull'aspirazione, da parte della nobiltà napoletana, a concorrere al governo attraverso l'inserimento nei seggi cittadini, e sulla sua stratificazione in età moderna, si veda Giovanni Muto, «I "Segni d'Honore”. Rappresentazioni delle dinamiche nobiliari a Napoli in età moderna", in M. A. Visceglia, a cura di, Signori, patrizi, cavalieri nelletà moderna, Laterza, Bari, 1992, pp. I7I-192; sugli episodi di conflitto delle famiglie nobili escluse dai seggi, si veda IDEM, "Gestione politica e controllo sociale nella Napoli spagnola», in C. de Seta, a cura di, Le città capitali, Laterza, Roma-Bari, 1985, pp. 77-79.

77. Colapietra, Vita pubblica, p. 8I.

78. L'espressione, insieme alla notizia della pretesa di un'orazione da parte del viceré, è ripresa da Natale VeSCIO, «Politica e istituzioni negli Affetti di un disperato di Giambattista Vico", Itinerari di Ricerca Storica, 2 (2017), pp. II3-I34. In questo saggio si accenna al rapporto tra il filosofo e il viceré, oltre ad analizzare l'opera di Vico e gli effetti sociali della campagna di intimidazione provocata dalla Curia napoletana.

79. "Qui, cum omnia 'de meliore nota' bonarum artium ac utilium scientiarum studia tam misere tanque foede iacerent, ut de nominis gloria, cui apud quamlubet exteram doctissimam nationem comparandae innumeri nostrum toto pectore, omni studio incumbembant, iam haec urbs, non sine querelae admixtis lacrymis, desperaret, optimum literarum generi favisti, egregiae civium laudi consuluisti, summae nostrae civitatis amplitudini providisti», Pro Auspicatissimo in Hispaniam reditu Francisci Be-

Pedralbes, 4I (202I), 255-298, ISSN: O2II-9587, DOI: IO.344/PEDRALBES2O2I.4I.8 
[...] a lui si offerse una bella occasione di promuoverlo: che '1 Signor D. Niccolò Caravita, per acutezza d'ingegno, per severità di giudizio e per purità di Toscano stile Avvocato Primario de' Tribunali e gran Favoreggiatore de' Letterati, volle fare una Raccolta di componimenti in lode del signor conte di Santostefano, viceré di Napoli, nella di lui dipartenza, la quale fu la prima che uscì in Napoli nella nostra memoria, e dentro le angustie di pochi giorni doveva ella essere già stampata. ${ }^{80}$

Il filosofo diventerà, pochi anni dopo, uno dei membri più illustri dell'Accademia Palatina, fondata nel 1698 dal duca di Medinaceli e composta anche da togati e aristocratici spagnoli già inseriti nell'apparato burocratico. ${ }^{81}$ La nuova cultura incontrava ancora una volta il favore vicerale ma inquadrandosi questa volta in maniera ufficiale e assicurandosi una protezione da un'eventuale nuova offensiva clericale. ${ }^{82}$

navidii S. Stephani Comitis Atque in Regno Neapolitano Pro Rege Oratio, in Nicola Caravita, Varj componimenti in lode dell'eccellent. signor D. Fr. Benavides viceré nel regno di Napoli raccolti da D. Nicolò Caravita, presso Giuseppe Roselli, Napoli, I696, p. 2. Sulle dediche a Benavides nei libri illustrati, si veda Rodney Palmer, "Viceregal dedicatees of Neapolitan Illustrated books», in J. Colomer, España y Nápoles, pp. 408-409. Si veda anche, per una bibliografia aggiornata sulla tradizione delle dediche ai viceré, Vidales del Castillo, «El vir Marqués del Carpio y las letras», p. 385, nota 1316.

80. Giambattista Vico, Vita di Giambattista Vico scritta da se medesimo, in Angelo Calogerà, Raccolta d'opusculi scientifici, e filologici, appresso Cristoforo Zane, Venezia, I728, vol. I, p. I89.

8I. Confuorto racconta che nel marzo del I698 «si è unita un'assemblea di circa venti tra titolati, cavalieri ed altre persone erudite, col titolo d'Accademia Reale, per discorrere in essa di materie di geografia e matematica», e che si stabilì il giovedì come giorno di incontro: Confuorto, Giornali, II, p. 298. Sulla genesi e struttura dell'Accademia, si vedano Lezioni dell'Accademia di Palazzo del duca di Medinaceli, a cura di M. Rak, Istituto Italiano per gli Studi Filosofici, Napoli, 2000-2003, Giuseppe RicuPERATI, «L'immagine della Spagna a Napoli nel primo Settecento: Vico, Carafa, Doria e Giannone», in A. Musi a cura di, Alle origini di una nazione. Antispagnolismo e identità italiana, Guerini e Associati, Milano, 2003, pp. 83-II2.

82. Galasso, Napoli spagnola, pp. 534-535.

Pedralbes, 4I (2O2I), 255-298, ISSN: O2II-9587, DOI: IO.344/PEDRALBES2O2I.4I.8 


\section{3. «Por favorecer la verdad»: il viceré Benavides e Francesco D'Andrea}

Tra i protagonisti del nuovo orientamento della cultura napoletana, il più attivo fu Francesco D'Andrea, che riuscì a trasferire i principi degli Investiganti nella vita civile, facendo compiere alla cultura giuridica italiana un grande passo in avanti, poiché considerava la strada dell'avvocatura «unica via d'acquistar nella nostra città, particolarmente a nobili fuor di piazza, onori, ricchezze, dignità et acquistate conservarle». ${ }^{83}$ Il suo percorso biografico e politico fu particolarmente difficile e accidentato, perché interamente attraversato dall'accusa ingiusta di essere stato uno dei responsabili della rivoluzione masanelliana. Ancora molti anni dopo, nel 1682, Antonio di Sangro, in una seduta del Sacro Regio Consiglio, lo apostrofava come «Masaniello capopopolo». ${ }^{84}$ Per difendersi dalle accuse e ribadire il proprio lealismo al potere regio, D'Andrea scrisse la Relazione de' servizi fatti dal signor Francesco di Andrea nel tempo che esercitò il posto di avvocato fiscale nella provincia di Abruzzo Citra. ${ }^{85}$

La calunnia affondava le sue radici nel 1646 , alla vigilia della rivoluzione, quando D'Andrea era stato nominato fiscale di Chieti dal viceré duca d'Arcos, ruolo che ricoprì fino al I648. Fu questo incarico che diede luogo all'accusa che circolò a lungo di «haver, né tumulti del 47, servendo d'Avocato fiscale nell'Audienza di Chieti, servito al Popolo». ${ }^{86}$

83. D'Andrea, Avvertimenti ai nipoti, p. 270. Sulla figura di D'Andrea e sul risveglio culturale, si veda anche la monografia di José María Marín, Castellanos viejos de Italia. El gobierno de Nápoles a fines del siglo XVII, Giuffré, Milano, 2003.

84. D'Andrea, Avvertimenti ai nipoti, p. 20, pp. 382-383, nota 592.

85. La Relazione de' servizi fatti nella provincia di Abruzzo Citra è rimasta in forma manoscritta ed è conservata in BNN, ms I7I.G.9; la notizia è in Stefano CAPONE, «Francesco D'Andrea e il rinnovamento culturale del Seicento a Napoli (in occasione del rinvenimento di un manoscritto sconosciuto degli Avvertimenti ai nipoti)", La Capitanata. Rassegna di vita e di studi della Provincia di Foggia, 34 (1997), p. 55.

86. Così scrisse il gesuita Giovanni Berardi alla segreteria di Stato del Granduca di Toscana, nell'estate del I679; la notizia è in D'Andrea, Avvertimenti ai nipoti, pp. 19-20. 
Questa nasceva dall'omonimia con un certo Francesco D'Andrea, fratello del noto Vincenzo, rivoluzionario e collezionista d'arte morto probabilmente nel 1652 a seguito di un avvelenamento.$^{87} \mathrm{Se}$ i due fratelli, entrambi «avvocati lunghissimo tempo et assai dotti», ${ }^{88}$ che avevano assunto il comando durante la seconda fase della rivoluzione, non avevano legami di parentela con l'omonimo accusato, non è del tutto chiaro in che rapporti fossero, poiché dall'analisi dei movimenti bancari dei tre D'Andrea sono emerse delle sovrapposizioni che lasciano spazio a dubbi. ${ }^{89}$ C'è anche da mettere in luce che Vincenzo, come lo stesso Francesco, era un membro dell'Accademia degli Oziosi, dove i dibattiti intellettuali avranno sicuramente incluso riflessioni sulle prime forme di indipendentismo, e che i due potrebbero aver comunque condiviso i principi della rivoluzione costituzionale..$^{\circ \circ}$

Tuttavia Francesco non fu solo perseguitato dal sospetto di aver contribuito ai moti, ma anche da quello di aver assunto, rispetto agli altri Investiganti, una posizione molto più estrema in materia di fede, pur non avendo mai pubblicato nessuna opera connessa alla sua esperienza di accademico. Dopo la chiusura dell'Accademia Colonnese, alla fine del I669, D'Andrea aveva infatti cominciato a viaggiare attraverso l'Ita-

87. Ivi, pp. 382-383, nota 592. Sul ruolo di Vincenzo D'Andrea come guida politica, si veda: Vittorio Conti, La Rivoluzione repubblicana a Napoli e le strutture rappresentative (I647-I648), Centro Editoriale Toscano, Firenze, 1984; Rosario Villari, Un sogno di libertà: Napoli nel declino di un impero 1585-1648, Mondadori, Milano, 2012, pp. 474477; Gibran Bautista y Lugo, «Constantia et clementia. El lenguaje de la gracia real en cuatro ciudades en rebeldía Quito, México, Nápoles, Barcelona I592-I652», Pedralbes. Revista d'Història Moderna, 40 (2020), pp. 2II-256. Sul ruolo di collezionista, si veda Vincenzo Pacelli, «La quadreria di Vincenzo D’Andrea, giurista e rivoluzionario, da un inventario del 1649", Ricerche sul '6oo napoletano, 1987, pp. I45-I5I.

88. D'Andrea, Avvertimenti ai nipoti, p. 275.

89. Ivi, p. 17, nota 22. Sui banchi napoletani usati da Vincenzo D'Andrea come strumento per portare avanti il suo programma ideologico, vedi Andrea Zappulli, «1647-I648: Il Banco dei Poveri tra repubblicanesimo e rivolta», Napoli nobilissima, 2018, pp. 5-15.

90. Si veda Aurelio Musi, «Non pigra quies. Il linguaggio politico degli accademici oziosi e la rivolta napoletana del I647-I648", in E. Pii, a cura di, Il linguaggio politico delle rivoluzioni in Europa, Olschki, Firenze, 1992, pp. 85-IO4.

Pedralbes, 4I (2O2I), 255-298, ISSN: O2II-9587, DOI: IO.344/PEDRALBES2O2I.4I.8 
lia, fino al 1675 , anno in cui ritornò a Napoli ma lavorando soprattutto come avvocato e non nell'amministrazione cittadina; erano gli anni del viceregno del marchese de Los Vélez, che stava garantendo un temporaneo avvicinamento tra la politica vicereale e le aspirazioni del ceto forense. D’Andrea rifiutò la carica di eletto del Popolo, nel I679, continuando a sostenere l'importanza vitale dell'autonomia del ceto civile ma poi, deluso ancora una volta dalla politica di controllo burocratico del viceré marchese del Carpio, caratterizzata da una linea "centrista» che già aveva caratterizzato il governo di Ońate e da un assolutismo amministrativo che favoriva la presenza di funzionari spagnoli e quindi l'affermazione dei «togati» rispetto agli avvocati, decise di trascurare gli incarichi pubblici per dedicarsi esclusivamente allo studio..$^{9 \mathrm{I}}$

Fu solo l'arrivo di Benavides a dargli la possibilità di tornare alla ribalta sulla scena politica partenopea. Il viceré, riconoscendogli le ampie competenze governative, l'aveva subito reclutato nell'amministrazione cittadina, prima come giudice della Vicaria, nel I688, poi come consigliere del Sacro Regio Consiglio nel 1689 e, un anno dopo, come avvocato fiscale della Sommaria, nomina accolta con «applauso universale». ${ }^{92} \mathrm{Il}$ riaccendersi delle ingiurie si fa risalire a quest'ultima carica, quando le terne per la nomina arrivarono al Consiglio d'Italia e il reggente Pedro Guerrero, rappresentante di quell'ambiente conservatore che tanto avversava l'adesione alla filosofia moderna, pose come ostacolo insuperabile il suo presunto passato rivoluzionario e la partecipazione a «una escuela como de Ateystas». ${ }^{93}$ Il Consiglio d'Italia discusse l'argomento, ma Guerrero continuò a opporsi, proponendo l'esclusione da qualun-

9I. La definizione è data da Musi, Limpero, p. 248. Sul governo napoletano del marchese del Carpio e sulla sua politica di sostegno ai «togati», portata avanti per contrastare i tentativi di autonomia di un settore abbastanza consistente della nobiltà, si veda Capone, «Francesco D’Andrea», p. 7I; Galasso, Napoli nel viceregno, pp. 552-554.

92. BSNSP, Avvisi, II aprile I690, num. 38. Anche Pietro Giannone si soffermò su questa nomina, in cui D'Andrea era riuscito a conciliare «l'erudizione, l'istoria e la vera Giurisprudenza colle disputazioni forensi»: Giannone, Istoria Civile, Iv, pp. 489-490.

93. Si veda D'Andrea, Avvertimenti ai nipoti, p. 24; il documento è conservato presso l'Archivo General di Simancas (AGS), Secretarias Provinciales, Nápoles, lib. 649 , f. IIо. 
que incarico amministrativo, così che si decise di sospendere la procedura e nominare una commissione d'inchiesta per chiarire le responsabilità di D’Andrea. Tuttavia la commissione non fu mai composta, proprio per la difficoltà di trovare persone in grado di giudicare in maniera neutrale e perché Carlo iI non aveva accolto l'iniziativa; il Consiglio gli conferì ugualmente la carica, non potendo escludere un personaggio di così grande cultura e preparazione solo sulla base di calunnie. $^{94}$

In merito a queste vicende e al passato sospetto di D'Andrea, alcune lettere ritrovate nell'Archivio di Medinaceli di Toledo e finora inedite, scambiate nel 1689 tra il viceré Benavides, il segretario spagnolo Manuel Francisco de Lira, il presidente del Sacro Regio Consiglio Feliz de Lanzina y Ulloa e il reggente del Collaterale Diego de Soria Morales, marchese di Crespano, forniscono ulteriori dettagli, mettendo in luce la vicinanza del viceré a D'Andrea e alla difesa della sua posizione.95

La prima missiva è quella di Manuel Francisco de Lira, indirizzata a Benavides e contenente un ordine di Carlo II, cioè la richiesta di un informe su Francesco D'Andrea. Carlo II aveva necessità di conoscere l'opinione di Benavides su «letras, prendas y capacidades del Don Francesco Andrea", con lo scopo di smentire o confermare alcune notizie relative alla sua partecipazione ai moti rivoluzionari del i647 e all'atteggiamento verso la Chiesa, con cui aveva una "causa pendiente». Il segretario sottolinea, riassumendo la situazione difficile in cui si trovava D’Andrea, che tutti i viceré, tranne il marchese del Carpio, che ignorava i suoi "defectos», lo avevano punito con «en non emplearle, ni ponerle en las nominas». Tutte queste informazioni il viceré poteva ottenerle «comunicando sobre todo con el Presidente del Sacro Regio Consejo» e con il marchese di Crespano. Questi ultimi, schierandosi a difesa di D'Andrea, risposero al viceré e al segretario spagnolo con due lunghe relazioni in cui argomentavano intorno ai temi oggetto di diffamazione. Entrambe attraversavano la sua complessa parabola politi-

94. D'Andrea, Avvertimenti ai nipoti, pp. 26-27.

95. ADM-To, leg. 79, ramo 2, 9 giugno-8 luglio I689, ff. 23-24, a cui si fa complessivamente riferimento per i successivi brani citati.

Pedralbes, 4I (2O2I), 255-298, ISSN: O2II-9587, DOI: IO.344/PEDRALBES2O2I.4I.8 
ca, facendo emergere, come scrive Feliz de Lanzina e Ulloa, la figura di un intellettuale accusato ingiustamente da due "obligaciones se le hagan terribles, pero cuantos más terribles menos creibles» e costretto a «irse a ver las ciudades de Italia».

Dalle parole del presidente del Sacro Regio Consiglio, risalta la figura di un intellettuale le cui «extravagancias» non erano altro che conseguenza di una "gran libertad de animo». Le stravaganze a cui si riferisce Lanzina e Ulloa risiedevano forse in quei due aspetti apparentemente contrastanti ma coesistenti nella persona di D'Andrea: l'adesione alla filosofia moderna e la promozione di una radicale riforma politica. La sua intelligenza e la sua profonda cultura mal si conciliavano, per i sostenitori dell'ambiente conservatore (rappresentati proprio da Guerrero e da coloro che volevano impedire la sua nomina a consigliere del Sacro Regio Consiglio), con la sua ribellione all'ordine prestabilito. Sempre nella relazione si ricordava inoltre che proprio in nome dell'adesione alle nuove correnti di pensiero era stata disposta, da parte delle autorità ecclesiastiche, l'espulsione di D'Andrea dall'Accademia:

Esta dicheria la a apoyado la emulación a una Academia que se tuvo en casa del marqués de Arena donde intervino con otros el Juez Francesco de Andrea, allí entre otras se disputó la questión de Anima Mundi, y otras ventiladas de los Filósofos antiguos más para obstentación del Ingenio, como se pude y debe creer, que porque se dudase de lo que ha determinado nuestra Señora la Santa Iglesia.

Così come infondato si era rivelato anche il sospetto relativo al suo coinvolgimento nei moti rivoluzionari, quando «en el tiempo de las revoluciones del 48 hubiese sido capapopulo; esto no es verdad ni tiene fundamento». Il Presidente del Sacro Regio Consiglio, arrivato a Napoli nel I650, quando ancora «duraba la inondación de la pasada tormenta", riferisce di aver scandagliato a fondo tutti i libri stampati e manoscritti a quel tempo in possesso degli intellettuali, senza tuttavia avervi mai trovato tracce di eresia in quelli di D'Andrea.

La stessa posizione era sostenuta dal marchese di Crespano, il quale aveva raccolto notizie «de personas de esta ciudad y de muchos de Abruzo», tra cui Cesare Pignatelli, duca di Rocca, che "no solo me lo 
refirió a boca pero me lo dio por escrito a que preste entiero credito por la calidad del caballero». Tutte le testimonianze gli permettevano di poter confermare l'estraneità di D'Andrea, ribadendo l'opinione positiva già espressa in una passata "relación que tocava a materia tan grave».

Alla luce di tutte queste informazioni, Benavides rispose al segretario de Lira con una relazione riassuntiva, sicuramente preliminare all'informe che non è stato purtroppo possibile rintracciare. Questa si apre con un grande segno di riconoscimento verso D'Andrea, definito intellettuale «del primer credito de literatura y toda Italia» e si chiude con un invito rivolto direttamente a Carlo II a prendere in considerazione l'opinione positiva su «un pobre hombre como este solo por favorecer la verdad, y en esto no me queda duda alguna». D'altronde Benavides stesso mette in evidenza di aver nominato D'Andrea giudice della Vicaria, ruolo in cui quest'ultimo aveva sempre dimostrato «juicio y puntualidad», a differenza di tanti altri «sujetos de muy inferior credito a este» che erano invece riusciti a occupare posti di rilievo nell'amministrazione cittadina, come ad esempio Lorenzo Giordano. Benavides infatti, costretto suo malgrado ad approvare le direttive reali, avrebbe dimostrato la sua perplessità per la nomina di Lorenzo Giordano, figlio del celebre pittore napoletano Luca, a presidente della Sommaria nel $1692,{ }^{96}$ visti i troppi ruoli già rivestiti nella burocrazia cittadina fin dal I688, così come avrebbe definito la proposta del ventiduenne Giovan Battista Pisacane a consigliere di Santa Chiara come un caso

96. Il viceré aveva infatti invitato, in una lettera diretta al segretario Juan de Angulo, alla cautela nel disporre questa nomina e ad affidarsi al Consiglio d'Italia per la decisione: si veda Maria Jesus MuÑoz González, «Documentos inéditos sobre la llegada a España de Luca Giordano», Ricerche sul '6oo napoletano. Saggi e documenti 2003-2004, Electa Napoli, 2004, p. I62. Carlo II gli concesse comunque la nomina, così come concesse numerose mercedes a molti membri della famiglia del pittore, ma fu in particolare per Lorenzo che si prodigò affinché percepisse regolarmente il mensile per le contemporanee cariche di giudice della Vicaria e presidente della Sommaria: si veda BNN, Ordini reali della Camera in lingua spagnola, ms. XI B (ff. 37v-38r; ff. I36v-I37). Sulle cariche occupate da Lorenzo, si veda anche InTORCIA, Magistrature, p. 318. 
«delli più gravi che mai siano occorsi in questa città».97 $\mathrm{Il}$ viceré sottolineava infatti la gravità della situazione napoletana, in cui a causa di nomine conferite frettolosamente o per "gusto del Re», troppe persone meritevoli restavano indietro, riferendosi sicuramente anche a D'Andrea, che proprio grazie a lui aveva potuto invece riavvicinarsi a quella politica vicereale da cui era rimasto deluso più volte. Il sostegno offerto a D'Andrea da parte di Benavides, anche grazie all'appassionata relazione diretta al segretario de Lira, può adesso essere considerato frutto di una scelta personale più che semplice specchio degli interessi politici della Corona. D'altronde è da considerare significativo che negli ultimi anni del Seicento furono emanate una serie di ordini volti allo snellimento dell'apparato burocratico, a Napoli così come in Catalogna e Castiglia, segno di una volontà di riforma che circolava fra gli amministratori spagnoli. ${ }^{98}$ Fu proprio il viceré Benavides, tra il I69I e il I692, a ordinare la riduzione del personale delle segreterie di Giustizia e Guerra, così come della Sommaria e della Vicaria. ${ }^{99}$ L'inserimento di D'Andrea nelle maglie della burocrazia si configura quindi ancora di più come una scelta mirata, una possibilità accordata all'avvocato di poter denunciare la corruzione degli uffici e suggerire concrete misure per affrontare alcuni problemi, come quello della riforma monetaria e della riorganizzazione dell'apparato amministrativo. Purtroppo nel I695, mentre si trovava a Procida, D’Andrea fu esonerato dagli incarichi: le sue idee avevano incontrato la resistenza del baronaggio e di quel settore del ceto togato che non aveva intenzione di veder turbato l'assetto corrotto di cui faceva parte, e neanche l'appoggio di Benavides, che lo

97. D'Andrea, Avvertimenti ai nipoti, p. 35 .

98. Sui meccanismi dell'assegnazione e della venalità degli uffici a Napoli in età moderna, si veda Roberto Mantelli, Il pubblico impiego nell'economia del Regno di Napoli: retribuzioni, reclutamento e ricambio sociale nell'epoca spagnuola (secc. XVI-XVII), Istituto Italiano per gli Studi Filosofici, Napoli, 1986, pp. 217-307. Sulla documentazione relativa agli uffici napoletani nella prima metà del XVII secolo, e conservata negli archivi napoletani, si veda Vittorio Ivo Comparato, Uffici e società a Napoli (I600-I647). Aspetti dell'ideologia del magistrato nell'età moderna, Olschki, Firenze, 1974.

99. Mantelli, Il pubblico impiego, p. I86. 
raggiunse sull'isola, riuscì a incidere molto. ${ }^{\text {100 }} \mathrm{Il}$ ministero togato stava vivendo infatti in quegli anni una forte lotta al suo interno: da una parte la corrente incline alle riforme e dall'altra quella opportunista e meno colta, preoccupata solo di conservare lo status quo e che faceva fronte comune con la nobiltà. ${ }^{\text {Ior }}$ Il fallimento dell'azione intrapresa da D'Andrea rese ancora più evidente questa frattura, rafforzando così proprio quelli che non avevano creduto nelle idee dell'avvocato.

Neppure l'orientamento moderno di Benavides, spinto chiaramente da una volontà di riforma, riuscì a influire positivamente sulla riuscita del programma deandreiano. Le sue idee in merito a un'armonia tra potere regio, politica vicereale e aspirazioni dei magistrati non ebbero seguito, mentre sul finire del secolo si stava delineando un equilibrio molto diverso tra le componenti sociali. ${ }^{\text {102 }}$

Nonostante ancora alcune zone d'ombra, da queste tormentate vicende sembra emergere il ruolo positivo di Benavides insieme alla ricca eredità culturale lasciata da D'Andrea. Una luce radente mette infatti in risalto non solo un viceré in cui la curiosità intellettuale si profilava come uno strumento di comprensione di una società in trasformazione, attraversata dai nuovi venti di pensiero che soffiavano dall'Europa, ma anche il forte ascendente di D’Andrea sulla nuova generazione. È indubbio che il ruolo di guida intellettuale dell'avvocato lasciò tracce indelebili, condizionando il pensiero dei protagonisti di inizio secolo: Serafino Biscardi e Gaetano Argento, fino ad arrivare a Pietro Giannone. ${ }^{\mathrm{IO}} \mathrm{Il}$ quadro politico in cui operarono i tre intellettuali era profondamente cambiato, e l'arrivo degli austriaci, nel I707, pose fine al lungo periodo di dominio spagnolo. Sotto il governo dei viceré austriaci, il Consiglio Collaterale perse di importanza, trasformandosi, insieme alle altre magistrature, in un organo esecutivo delle direttive di Vienna, e la reazione anticuriale

Ioo. Capone, «Francesco D'Andrea», p. 58.

IoI. Sulla crisi del ceto togato, si vedano le riflessioni di Rovito, Il viceregno spagnolo.

IO2. CAPONE, «Francesco D’Andrea», p. 74.

I03. Giuseppe Ricuperati, La città terrena di Pietro Giannone: un itinerario tra crisi della coscienza europea e illuminismo radicale, Olschki, Firenze, $200 \mathrm{.}$.

Pedralbes, 4I (2O2I), 255-298, ISSN: O2II-9587, DOI: IO.344/PEDRALBES2O2I.4I.8 
che aveva animato fino ad allora la città napoletana, restò viva solo per un breve periodo. ${ }^{\mathrm{I} 4} \mathrm{La}$ nomina del curialista cardinale Friedrich Michael Althmann (I722-I728), segnò infatti l'inizio di una fase discendente nella polemica; il nuovo viceré adottò una politica di complicità con la Santa Sede, ostacolando tutte le iniziative politiche che si ponevano in netto contrasto con la sua linea. Eppure la promozione della nuova cultura non si era affatto arrestata. Furono quelli gli anni in cui Gaetano Argento, con la sua dissertazione De re beneficiaria, concepita tra il I707 e il I709, difendeva dalle ingerenze romane la Real Giurisdizione, sancendo il passaggio dalla tematica antifeudale a quella giurisdizionalista, ${ }^{\text {105 }}$ Pietro Giannone analizzava, nell' Istoria civile del regno di Napoli, pubblicata nel I723, i rapporti tra Stato e Chiesa, ribadendo la condanna dei metodi adottati dal Sant'Ufficio, e Giambattista Vico pubblicava, nel I725, la sua opera più fortunata, La Scienza Nuova.

Agli albori del xvin secolo e fino alla piena affermazione dell'Illuminismo partenopeo, la lezione moderna di D'Andrea non era quindi stata accantonata e continuava ad affondare tenacemente le sue radici nella realtà napoletana, traducendo in concretezza lo spirito di rinnovamento della sua generazione.

\section{Appendice}

Lettere tra Manuel Francisco de Lira, Feliz de Lanzina y Ulloa, Francisco de Benavides e Diego de Soria Morales (Madrid - Napoli, 9 giugno-8 luglio 1689). ${ }^{\text {106 }}$

IO4. IDEM, "Napoli e i Viceré austriaci 1707-1734", in Storia di Napoli, Napoli, 1976, vol. IV, pp. 383-385.

I05. Sul delicato passaggio alla conquista austriaca e sulla posizione dell'intellettuale Argento, la cui dimora divenne il luogo d'incontro dei maggiori intellettuali del tempo, tra cui Giannone e Vico, si veda Elvira Gencarelli, s.v. «Argento, Gaetano», in Dizionario Biografico degli Italiani, Istituto dell'Enciclopedia Italiana, Roma, 1962, vol. 4.

Io6. Nella trascrizione delle lettere, così come in tutti i passi riportati nell'articolo, si è provveduto a rispettare criteri conservativi, provvedendo solo a sciogliere le forme 
Archivo de Medinaceli-Toledo, leg. 79, ramo 2, ff. 23-24.

Manuel Francisco de Lira a Francisco de Benavides:

Excelentisimo Señor. Hallase su Majestad con encontrados informes de las letras, prendas y capacidad del Don Francesco Andrea pues por una parte se supone que procede con aprovación en la Plaza que ocupa de Juez de la Gran Corte de la Vicaria; que es de los mayores letrados que hay en Italia como lo comprueban los papeles y libros que ha dado a la estampa; y por otra que concurren en su persona capitales defectos; que la caveza es turbida y variable; que siendo Fiscal de la Audiencia de Chiete en tiempo de las revoluciones fue como de los sediciosos; que en un informe que hizo el Señor Marqués de los Vélez el año de 1678 dijo que el Obispo de Gravina Inquisidor Apostolico le había dicho le seguía los pasos, y en tribunal tenía causa pendiente; que aunque el año de I682 el mismo Marqués de los Vélez recomendó a Su Majestad este sujeto con una relación del de Crespano fue fiandose este de papeles que le presentò el interesado; y finalmente que los virreyes no le han castigado por el indulto general, contentandose en no emplearle, ni ponerle en las nominas hasta en tiempo del Señor Marqués del Carpio que sin duda ignorava sus defectos. En esta variedad de noticias quiere su Majestad saber lo cierto, y me manda que reservadamente diga a Vuestra Excelencia informe por esta via de las más fundamentales que adquiriere, y llamando Vuestra Excelencia al Marqués de Crespano sepa Vuestra Excelencia del que papeles vió de este maestro para el informe que hizo de sus procedimientos al Señor marqués de los Vélez, y expecialmente de lo que obró en las revoluciones de ese Reyno, y que confiando y comunicando Vuestra Excelencia sobre todo con el Presidente del Sacro Consejo diga Vuestra Excelencia con todo secreto, y reserva lo que se le ofrece de las operaciones de Don Francesco por lo pasado, y de la forma en que se porta al presente y el verdadero concepto en que Vuestra Excelencia se alla, y hiciese [...] de la firmeza de su juicio con todo lo demás que Vuestra Excelencia [...] conduce á que Su Majestad esté enterado de todas las propiedades de este sujeto. Guarde Dios a Vuestra Excelencia felices años como desea.

Madrid, 9 de junio I689.

abbreviate. Vengono indicate con [...] parole non chiaramente leggibili a causa della grafia indecifrabile o danneggiamento del testo; il simbolo $\left(^{* * *}\right)$ denuncia le cancellature, mentre le parti ritenute di scarso interesse vengono segnalate con $[\ldots]$.

Pedralbes, 4I (2O2I), 255-298, ISSN: O2II-9587, DOI: IO.344/PEDRALBES2O2I.4I.8 
Feliz de Lanzina y Ulloa a Francisco de Benavides (copia):

Excelentisimo Señor. Mandame Vuestra Excelencia que para satisfacer aun orden de Su Majestad (que Dios guarde) diga que siento de la persona del Juez de Vicaria Don Francesco de Andrea y por lo que toca a la literatura, ninguno ha dificultado que sea de la mejor no solo de este Reyno pero de toda Italia. Esto sus emulos no se tienen a negarlo, pero para degradar su gran talento, an procurado oponerle tadras (?), no ordinaría sino tales que aunque fuese el talento mayor aún, quedase de ellas superado. Desacreditan el juicio notandolo de ligero, y incostante por algunas licencias que ha permitido a su genio. Lo que en esto he observado en muchos años que le conozco es que a manifestado condición fuerte y libre que lo a tirado a poner en ejecución algunas resoluciones que abemos notado de estravagantes todos, pero no de calidad que perjdiquen a ninguno, tocasen en delito, o pasasen a ofender el honesto porque sus extravagancias no sé que avían ofendido con ninguno ni que aya cometido exceso porque se alle inquisido ni pecado que aia escandalizado antes con vivir celibe a vivido y vive bien honestamente todo lo que he excedido es efecto de una gran libertad de animo, como fue la resolución que tomó pocos años ha, de cuando estaba cargado de las maiores causas de estos Tribunales, sin embarazarle ni el interés de lo que le frutaban y podran frutar, ni el desconsuelo y respecto de sus clientes, se resolució a dejar las todas, y irse a ver las ciudades de Italia bastantemente dichoso es el que de los defectos está sujeto a los menores, lo que en grandes ingenios aun es más de alabar, o más de dispensar o compadecer; pero aun este defecto después que ha entrado en la religión del Ministerio le a correcido de manera que es ejemplo y puede ser dechado de [...] de flema de aplicación de circunspección puntualidad y pureza. Dos otras obligaciones [...] se le agan terribles pero quantos más terribles menos creibles. La primera es de que se dude si creé y siente bien de nuestra feé catolica porque se suponga que la Inquisición lo tenga en nota. Lo que sabemos y vemos es que vive como vivimos todos los Catholicos, y que observa lo que observamos todos. Esta dicheria la apoyado la emulación a una Academia que se tuvo en casa del Marqués de Arenas donde intervino con otros el Juez Francesco de Andrea, allí entre otras se disputó la questión de Anima Mundi, y otras de las ventiladas de los Philósofos antiguos más para obstentación del ingenio, como se pude y debe creer, que porque se dudase de lo que a determinado nuestra [...] la Santa Iglesia acuerdome que entonces lo que no interveníamos los motivábamos a los academicos y les decíamos que se tubiesen fuertes que quanto antes andarían a la Inquisición a responder 
quid sentirent de fide Dio escandalo esta Academia se dispusó su dismisión y no se a ablado mas; y esta sospecha hace solo a guerra a Francesco de Andrea, y no a tantos otros como intervinieron en aquella Academia. La segunda in humanis (?) estan bien la más honrada y es que en el tiempo de las revoluciones del 48 hubiese sido capapopulo: esto no es verdad ni tiene fundamento. Yo bine a este Reyno el año de I650 que aun duraba la inondación de la pasada tormenta, y para saber de quienes me debía guardar [...] hice muchas dilingencias informandome y recojiendo libros estampados y manuscritos, y aunque en ellos noté muchos, y en aquellos tiempos no fueron buenes, de Francisco de Andrea no alle nada antes en Thomas de Santis que hizo la historia de aquellos accidentes que haze memoria de el y no le da esta tacha que si la tuviera no se la hubiera disimulado, como no la disimulo a otro personajes de maior esphera, después porque sus emulos le achacaban de esto, hizo una respuesta que a todos los Ministros de su Magestad nos la dió, en que $[. .$.$] no solamente aquella notta que en esto poco tenía que hacer; sino que$ dió a conocer quan bien y puntualmente abía servido a Su Majestad en aquellos tiempos y a mi me pareció que lo había probado y así nunca lo tube por sospechoso y lo he con gusto admitido y experado afectuoso y inclinado a los nuestros, que es quanto puedo representar a Vuestra Excelencia cuia Persona guarde nuestro Dios los largos ańos que le suplico. Julio 7 de i689 [...].

Don Felix de Lancinary Ulloa

Francisco de Benavides a Manuel Francisco de Lira:

Señor mio. De lo que su Majestad me manda en carta de Vuestra Señoria de 9 de junio acerca de lo que entiendo de las prendas, juicio y procedimientos del Don Francesco de Andrea, diré primeramente, que habiendo de proveer yo la primera Plaza de Vicaria en este Reyno, hize vanidad de darla a este sugeto, por el del primer credito de literatura y talento de toda Italia, y que tuvieron todos [...] que me quiso saber el admitirla, haviendo pasado a las del Consejo y Camara [...] sugetos de muy inferior credito a este. Después le propuse a su Majestad para Plaza del Consejo y llegando a essa corte la nomina, ohí el escandalo que había hecho la memoria de un hombre de tan graves tachas como las que se decía tener este, y saviendo [...] corrido de ignorar las [...] informe de algunos sugetos de mi satisfación, que me aseguraron todos lo mismo que contienen los informes adjuntos del Presidente del Consejo y el Marqués de Crespano este funda con razones tan seguras la aprovación que hizo del sujeto en tiempo del Marqués de los Vélez que no deja que dudar de 
su lealtad, la misma assegura el Presidente, y diré individualmente la Academia de erudición que motivó se ablase de que era en este teniendolo por una cossa ridícula y dechasco (?) quedaban a los que asistían a la Academia ( ${ }^{* * *}$ y ańade el huicio y puntualidad que muestra en el exercicio de su Plaza de que yo soy también testigo de manera que a Francesco de Andrea le tengo yo por mucho más dichoso que si ubiera conseguido la Plaza en la gloria deberse a los Presidentes su Majestad aprovado con tan grandes encomios de un Virrey de Nápoles y los dos primeros y más acreditados Ministros del Reyno particularmente quando estan seguro que conseguirá el premio de mano de un Rey tan justo que se acuerda de un pobre hombre como este solo por favorecer la verdad $\left({ }^{* *}\right)$ que quanto en la materia puedo decir a Vuestra Señoria a quien guarde Dios muchos ańos como deseo.

Nápoles 8 de julio I689 [...].

Diego de Soria Morales a Manuel Francisco de Lira:

Excelentisimo Señor, haviendo satisfecho mi obediencía pocos días ha al precepto que se sirvió de darme Vuestra Excelencia, a fín que le representase mi sentir acerca de la doctrina y prudencia del Don Francesco de Andrea abogado en estos Consejos y al Presidente Juez Civil de la Gran Corte de la Vicaria; y sobre todo del concepto en que le tenía de fidilidad, y amor en el servicio del Rey Nuestro Señor y de como se había portado en tiempo de las revoluciones populares de este Reyno el año de I647 y el siguiente que se hallaba empleado en la Fiscalía de la Real Audiencia de Abruzo Citra, con manejo de comisiones y negocios particulares represente a Vuestra Excelencia lo mismo que con consulta mia y de orden del Señor Marqués de los Vélez siendo Virrey de este Reyno, puse en su consideración; pero porque a lo referido me manda Vuestra Excelencia ańada el fín [...] en que apoie una relación que tocava a materia tan grave represento de nuevo a Vuestra Excelencia que no solo me movió por los papeles que se me presentaron los quales paran aun en mi poder sino en informes secretos, que procure adquirir de personas de esta ciudad y de muchas de Abruzo o que habían estado allí en tiempo de las revoluciones y después, y el uno de ellos fue Don Cessar Pińatelo Duque de la Rocca, que havía a su todo [...] siempre a Don Miguel Suthio, que se halló governando la referida Provincia de Abruzo Citra en tiempo de las revoluciones, como lo continuó con actos peligrosos, hasta que se huvieron sosegado, el qual me dijo que siempre havía tratado y confiado su thío del otro Don Francisco de Andrea, valiendose de el en las mismas materias de las revolucio- 
nes, y en otras criminales muy graves y civiles de grande interes de su Majestad y del patrimonio del arrendamento de la sal, con cuio dinero que le entregó el Don de Andrea se había mantenido el y la gente que le asistía, y que estava en las Plazas que se conservavan por su Magestad todo lo qual el dicho Don Cesar Pińatelo, no solo me lo refiriò a boca pero me lo dio por escrito a que preste entiero credito por la calidad del caballero y porque [...] comprobado el que se avía tenido del otro sugeto, pues sosegados los tumultos su Alteza el Señor Don Julio y el Señor Conde de Oñate le havían mantenido en el exercicio de la misma fiscalía por otros seis meses hasta que fue a servirla el proprietario a quien Su Magestad avía hecho merced de ella [...] a 8 de julio $1689[\ldots]$

El Marqués de Crespano 